HIM-2013-03

$\mathrm{MITP} / 13-029$

\title{
The pion vector form factor from lattice QCD and NNLO chiral perturbation theory
}

\author{
Bastian B. Brandt ${ }^{a}$, Andreas Jüttner ${ }^{b}$ and Hartmut Wittig ${ }^{c, d}$ \\ ${ }^{a}$ Institut für theoretische Physik, University of Regensburg, D-93040 Regensburg, Germany \\ ${ }^{b}$ School of Physics and Astronomy, University of Southampton, Southampton SO17 1BJ, \\ United Kingdom \\ ${ }^{c}$ PRISMA Cluster of Excellence, Institut für Kernphysik, University of Mainz, D-55099 \\ Mainz, Germany \\ ${ }^{d}$ Helmholtz Institute Mainz, University of Mainz, D-55099 Mainz, Germany
}

\begin{abstract}
We present a comprehensive study of the electromagnetic form factor, the decay constant and the mass of the pion computed in lattice QCD with two degenerate $\mathrm{O}(a)$-improved Wilson quarks at three different lattice spacings in the range $0.05-0.08 \mathrm{fm}$ and pion masses between 280 and $630 \mathrm{MeV}$ at $m_{\pi} L \geq 4$. Using partially twisted boundary conditions and stochastic estimators, we obtain a dense set of precise data points for the form factor at very small momentum transfers, allowing for a model-independent extraction of the charge radius. Chiral Perturbation Theory (ChPT) augmented by terms which model lattice artefacts is then compared to the data. At next-to-leading order the effective theory fails to produce a consistent description of the full set of pion observables but describes the data well when only the decay constant and mass are considered. By contrast, using the nextto-next-to-leading order expressions to perform global fits result in a consistent description of all data. We obtain $\left\langle r_{\pi}^{2}\right\rangle=0.481(33)(13) \mathrm{fm}^{2}$ as our final result for the charge radius at the physical point. Our calculation also yields estimates for the pion decay constant in the chiral limit, $F_{\pi} / F=1.080(16)(6)$, the quark condensate, $\Sigma_{\overline{\mathrm{MS}}}^{1 / 3}(2 \mathrm{GeV})=261(13)(1) \mathrm{MeV}$ and several low-energy constants of $\mathrm{SU}(2) \mathrm{ChPT}$.
\end{abstract}

Keywords: Chiral Perturbation Theory; Lattice QCD; 


\section{Contents}

1 Introduction

2 Computational strategy 3

2.1 Euclidean correlation functions . . . . . . . . . . . . . . . . 4

2.2 The pion form factor, the decay constant and the light quark mass . . . . . . . 5

2.3 Ratios of correlation functions and excited states . . . . . . . . . . . . 7

3 Lattice simulation and results

3.1 Simulation parameters . . . . . . . . . . . . . . . . . 7

3.2 Data analysis and fitting procedure . . . . . . . . . . . . . 9

3.2 .1 Pion mass and decay constant $\ldots \ldots \ldots \ldots$

3.2 .2 Form factor . . . . . . . . . . . . . . . . . 11

3.3 Finite volume corrections . . . . . . . . . . . . . . . . . . . 11

3.4 Extraction of the charge radius . . . . . . . . . . . . . . . . 12

4 Chiral and continuum extrapolations 14

4.1 Fits guided by chiral perturbation theory . . . . . . . . . . . . . . . 14

$4.1 .1 \quad$ NLO chiral fits . . . . . . . . . . . . . . . . . . 16

4.1 .2 NNLO chiral fits . . . . . . . . . . . . . . . . . 18

4.1.3 NLO and NNLO chiral fits: Final results . . . . . . . . . . . . . 21

4.2 Fits guided by polynomial models . . . . . . . . . . . . . . . . . . 21

4.3 Results at the physical point . . . . . . . . . . . . . . . 22

5 Conclusions and outlook $\quad 26$

A Appendix $\quad \mathbf{2 8}$

A.1 Chiral perturbation theory to NNLO . . . . . . . . . . . . . . . 28

A.2 Reformulation of ChPT to NNLO for global fits . . . . . . . . . . . . . 30

A.2.1 Conventions and necessary replacements . . . . . . . . . . 30

A.2.2 Reformulated formulae . . . . . . . . . . . . . . . 31

A.2.3 Inclusion of lattice artefacts . . . . . . . . . . . . . . . 31

\section{Introduction}

Thanks to the continued progress in improving numerical and field theoretical techniques [1 8$]$, computer simulations of QCD on a Euclidean space-time lattice are sufficiently advanced to produce reliable results for a number of phenomenologically important quantities (see for 
example the FLAG-summary [9]). Some of these results are postdictions which can serve as a test of lattice QCD as a tool, other results are real predictions which can be used to address the validity of the Standard Model. In both cases the estimation of systematic uncertainties is a crucial but often delicate issue.

In the case at hand, i.e. the pion electromagnetic form factor, the dominant systematic in recent calculations $10-15$ is due to its strong quark-mass dependence which complicates the extrapolation from unphysically heavy quark masses to the physical point. Chiral perturbation theory can provide guidance here. The corresponding expressions for the form factor as a function of the quark mass have been worked out up to NNLO [16 19]. However, concerning the effective theory's validity, a particular concern here is the tree-level contribution of vector degrees of freedom which can couple to the probing photon. In the effective theory these have been integrated out and enter only passively through the low-energy parameters in the effective Lagrangian. The scale separation between the Goldstone bosons $(\pi, \mathrm{K}, \eta)$ and the vector bosons $(\rho, \omega)$ is, however, not large, and one may be worried about the applicability of the low-energy effective theory. Other interesting observables like the hadronic vacuum polarisation do also receive tree-level contributions from vector particles, and similar concerns can be raised $20[24]$.

From a lattice practitioners point of view the mere evaluation of the pion form factor on a given lattice ensemble is a rather straightforward task, and therefore this quantity serves as an ideal laboratory for studying the above questions. Our strategy is to compare lattice QCD results to the predictions of chiral effective theory for the pion form factor and charge radius, the pion decay constant and its mass. For the pion mass and decay constant the expressions of $\mathrm{ChPT}$ are known to provide a good description of lattice data in the range of quark masses studied in this work (see $[9]$ ). In the same spirit we have concentrated on producing data for the form factor for very small space-like photon momenta, in order to remain in the realm of chiral perturbation theory. To this end we made extensive use of partially twisted fermionic boundary conditions [25 28, which allowed us to induce small pion momenta despite simulating in a finite lattice volume. Thereby we were able to determine the pion charge radius in a quasi model-independent way. By comparing a variety of fit ansätze based on Chiral Perturbation Theory (ChPT) at NLO and NNLO respectively, we investigated whether different fits provide a consistent description of the data and lead to reliable results for the pion charge radius and decay constant. For our final estimates we have performed an elaborate analysis of systematic uncertainties arising from lattice artefacts and finite-volume effects. As a byproduct we have determined the relevant low-energy constants (LECs) of two-flavour QCD at NNLO.

We briefly anticipate the core results: On a qualitative level we note that a joint description of our data for the pion mass, decay constant and form factor in terms of ChPT at NLO fails, while a consistent description of all three quantities in terms of ChPT can only be achieved at NNLO. We stress that the validity of this statement must be monitored as the pion mass is further decreased. In fact, our findings emphasise the importance of performing simulations at or very near the physical point. 
Our final results for decay constants, LECs and the charge radius are

$$
\begin{array}{lllll}
F_{\pi} & =90(8)(2) \mathrm{MeV}, & F & =84(8)(2) \mathrm{MeV}, & \\
F_{\pi} / F & =1.080(16)(6), & \Sigma^{1 / 3}=261(13)(1) \mathrm{MeV}, & \text { from NLO fit }, \\
\bar{\ell}_{3} & =3.0(7)(5), & \bar{\ell}_{4}=4.7(4)(1), & \\
& & & & \\
\left\langle r_{\pi}^{2}\right\rangle & =0.481(33)(13) \mathrm{fm}^{2}, & & & \text { from NNLO } \\
\bar{\ell}_{6} & =15.5(1.7)(1.3), & & &
\end{array}
$$

where the quark condensate $\Sigma$ is defined in the $\overline{\mathrm{MS}}$-scheme at a renormalisation scale of $2 \mathrm{GeV}$.

The outline of this paper is as follows: In section 2 we introduce the basic definitions and our computational setup. Simulation details and lattice results are presented in section 3 , followed by their discussion in terms of fits and extrapolations in section 4. Our conclusions are presented in section 5. Preliminary reports of the results included in this paper have appeared in 15,29 31.

\section{Computational strategy}

In this section we define the pion decay constant $F_{\pi}$, the pion mass $m_{\pi}$ and the pion charge radius $\left\langle r_{\pi}^{2}\right\rangle$ in terms of Euclidean two- and three-point functions.

The electromagnetic form factor in two-flavour QCD is defined by

$$
\left\langle\pi^{+}\left(\boldsymbol{p}_{f}\right)\left|\frac{2}{3} \bar{u} \gamma_{\mu} u-\frac{1}{3} \bar{d} \gamma_{\mu} d\right| \pi^{+}\left(\boldsymbol{p}_{i}\right)\right\rangle=\left(p_{f}+p_{i}\right)_{\mu} f_{\pi \pi}\left(q^{2}\right)
$$

where $q^{2}=\left(p_{f}-p_{i}\right)^{2}$ is the space-like momentum transfer, $-q^{2} \equiv Q^{2} \geq 0$. Near vanishing momentum transfer, the form factor can be expanded in powers of $q^{2}$. By convention, the linear term defines the charge radius, $\left\langle r_{\pi}^{2}\right\rangle$, i.e.

$$
f_{\pi \pi}\left(q^{2}\right)=1-\frac{1}{6}\left\langle r_{\pi}^{2}\right\rangle q^{2}+\mathrm{O}\left(q^{4}\right), \quad\left\langle r_{\pi}^{2}\right\rangle=\left.6 \frac{\mathrm{d} f_{\pi \pi}\left(q^{2}\right)}{\mathrm{d} q^{2}}\right|_{q^{2}=0}
$$

Simulations of lattice QCD are necessarily performed in a finite volume, and hence the accessible range of momentum transfers is rather limited. In a conventional setting (periodic fermionic boundary conditions) the initial and final pions can only assume the Fourier momenta, $\boldsymbol{n} 2 \pi / L$, where $\boldsymbol{n}$ is a vector of integers. Unless one can afford to simulate very large box sizes $L$, the lowest non-zero value of $Q^{2}$ can be rather sizeable. It is then doubtful whether the charge radius can be determined in a model-independent fashion, e.g. from the linear slope of the form factor near vanishing $Q^{2}$.

Partially flavour-twisted boundary conditions $25,28,32$ have by now become a standard tool to overcome this problem. By imposing periodicity on the quark fields in the spatial directions up to a phase factor, i.e.

$$
\psi\left(x+\hat{\boldsymbol{e}}_{j} L\right)=\psi(x) e^{i \theta_{j} / L} \quad j=1,2,3,
$$

it was shown in 12 that the momentum transfer satisfies

$$
-Q^{2} \equiv q^{2}=\left(p_{f}-p_{i}\right)^{2}=\left[E_{\pi}\left(\boldsymbol{p}_{f}+\frac{\boldsymbol{\theta}_{f}}{L}\right)-E_{\pi}\left(\boldsymbol{p}_{i}+\frac{\boldsymbol{\theta}_{i}}{L}\right)\right]^{2}-\left[\left(\boldsymbol{p}_{f}+\frac{\boldsymbol{\theta}_{f}}{L}\right)-\left(\boldsymbol{p}_{i}+\frac{\boldsymbol{\theta}_{i}}{L}\right)\right]^{2} .
$$


Here, $\boldsymbol{\theta}_{i}$ and $\boldsymbol{\theta}_{f}$ denote the vectors of twist angles applied to the quark probed by the electromagnetic current in the initial and final pion, respectively, and

$$
E_{\pi}(\boldsymbol{p})=\sqrt{m_{\pi}^{2}+\boldsymbol{p}^{2}}
$$

is the pion dispersion relation 32 . In this work we have paid particular attention to choosing twist angles which result in a very dense set of data points in the immediate vicinity of $Q^{2}=0$, such that the charge radius could be determined by means of a discretised derivative of the form factor.

\subsection{Euclidean correlation functions}

All our calculations have been performed in two-flavour QCD, employing $\mathrm{O}(a)$ improved Wilson fermions [33]. We have used the non-perturbative determination [34] of the improvement coefficient $c_{\mathrm{sw}}$ which multiplies the Sheikholeslami-Wohlert term.

In this work we consider correlation functions of the non-singlet, $\mathrm{O}(a)$ improved axial current and pseudoscalar density 35,

$$
\begin{aligned}
A_{\mu}^{I}(x) & =\bar{u}(x) \gamma_{\mu} \gamma_{5} d(x)+a c_{\mathrm{A}} \tilde{\partial}_{\mu} P(x), \\
P(x) & =\bar{u}(x) \gamma_{5} d(x),
\end{aligned}
$$

as well as the $\mathrm{O}(a)$ improved electromagnetic current, i.e.

$$
V_{\mu}^{I}(x)=V_{\mu}(x)+a c_{\mathrm{V}} \tilde{\partial}_{\nu} T_{\mu \nu}(x) .
$$

Since we are simulating mass-degenerate light quarks it is sufficient to consider only the local vector and tensor currents $V_{\mu}(x)=\bar{d}(x) \gamma_{5} u(x)$ and $T_{\mu \nu}(x)=i \bar{q}(x) \sigma_{\mu \nu} q(x)$, respectively. In the above expressions, $\tilde{\partial}_{\mu}$ is the symmetrised discrete derivative in direction $\mu$. The improvement coefficient $c_{\mathrm{A}} \equiv c_{\mathrm{A}}\left(g_{0}\right)$ has been computed non-perturbatively in two-flavour QCD in 36 for a range of bare couplings $g_{0}$ (see also table 1 ) and $c_{\mathrm{V}}$ has been computed in one-loop perturbation theory [37] but at the end of this section we will argue that it is of little relevance here. Note that the hadronic matrix elements of the currents must still be renormalised in order to yield physical observables.

We compute the following two-point functions

$$
\begin{aligned}
& C_{P P}(t, \boldsymbol{p})=\sum_{\boldsymbol{x}} e^{i \boldsymbol{p} \cdot \boldsymbol{x}}\left\langle P(t, \boldsymbol{x}) P^{\dagger}(0, \mathbf{0})\right\rangle \simeq \frac{\left|\mathcal{Z}_{P}\right|^{2}}{2 E(\boldsymbol{p})}\left(e^{-E(\boldsymbol{p}) t}+e^{-E(\boldsymbol{p})(T-t)}\right) \\
& C_{P A}(t, \boldsymbol{p})=\sum_{\boldsymbol{x}} e^{i \boldsymbol{p} \cdot \boldsymbol{x}}\left\langle P(t, \boldsymbol{x})\left(A_{0}^{I}\right)^{\dagger}(0, \mathbf{0})\right\rangle \simeq \frac{\mathcal{Z}_{P} \mathcal{Z}_{A}^{*}}{2 E(\boldsymbol{p})}\left(e^{-E(\boldsymbol{p}) t}-e^{-E(\boldsymbol{p})(T-t)}\right),
\end{aligned}
$$

where $T$ denotes the temporal extent of the lattice and where we have already indicated the asymptotic behaviour for large Euclidean time separations with the ground state matrix elements

$$
\mathcal{Z}_{P}=\langle 0|P(0)| \pi(\boldsymbol{p})\rangle, \quad \mathcal{Z}_{A}=\left\langle 0\left|\left(A_{0}^{I}\right)(0)\right| \pi(\boldsymbol{p})\right\rangle
$$


The electromagnetic form factor is extracted from the three-point function

$$
\begin{aligned}
& C_{3}\left(t, t_{f}, \boldsymbol{p}_{i}, \boldsymbol{p}_{f}\right)=\sum_{\boldsymbol{x}_{f}, \boldsymbol{x}} e^{i \boldsymbol{p}_{f} \cdot\left(\boldsymbol{x}_{f}-\boldsymbol{x}\right)} e^{i \boldsymbol{p}_{i} \cdot \boldsymbol{x}}\left\langle P\left(t_{f}, \boldsymbol{x}_{f}\right) V_{0}^{I}(t, \boldsymbol{x}) P^{\dagger}(0, \mathbf{0})\right\rangle \\
& \simeq \frac{\left|\mathcal{Z}_{P}\right|^{2}}{4 E\left(\boldsymbol{p}_{i}\right) E\left(\boldsymbol{p}_{f}\right)}\left\langle\pi\left(\boldsymbol{p}_{f}\right)\left|V_{0}^{I}(0)\right| \pi\left(\boldsymbol{p}_{i}\right)\right\rangle \\
& \quad \times\left[\Theta\left(t_{f}-t\right) e^{-E\left(\boldsymbol{p}_{i}\right) t-E\left(\boldsymbol{p}_{f}\right)\left(t_{f}-t\right)}-\Theta\left(t-t_{f}\right) e^{-E\left(\boldsymbol{p}_{i}\right)(T-t)-E\left(\boldsymbol{p}_{f}\right)\left(t-t_{f}\right)}\right] .
\end{aligned}
$$

In these formulae the initial pion source is located on the first timeslice, while $t$ is the temporal position of the insertion of the vector current and $t_{f}$ corresponds to the position of the sink.

The calculation of matrix elements of the $\mathrm{O}(a)$ improved vector current in the presence of twisted boundary conditions merits special attention, due to the presence of the derivative of the tensor current in eq. (2.8). The contribution from the term proportional to $c_{\mathrm{V}}$ to the three-point correlation function in eq. 2.11) reads

$$
C_{\mathrm{PTP}}\left(t, t_{f}, \boldsymbol{p}_{i}, \boldsymbol{p}_{f}\right)=a \sum_{\nu=0}^{3} \tilde{\partial}_{\nu} \sum_{\boldsymbol{x}_{f}, \boldsymbol{x}} e^{i \boldsymbol{p}_{f} \cdot \boldsymbol{x}_{f}} e^{-i\left(\boldsymbol{p}_{f}-\boldsymbol{p}_{i}\right) \cdot \boldsymbol{x}}\left\langle P\left(t_{f}, \boldsymbol{x}_{f}\right) T_{0 \nu}(t, \boldsymbol{x}) P^{\dagger}(0, \mathbf{0})\right\rangle,
$$

where $x_{f}=\left(t_{f}, \boldsymbol{x}_{f}\right)$ and $x=(t, \boldsymbol{x})$. Since $T_{00} \equiv 0$ only spatial derivatives yield non-vanishing contributions. Moreover, when periodic boundary conditions are imposed in the spatial directions, the discretised derivatives vanish exactly, owing to translational invariance.

In the presence of twisted boundary conditions this is no longer true. The appearance of phase factors implies that uncancelled contributions from the spatial boundary arise. A few lines of algebra then yield the expression for $C_{\mathrm{PTP}}$, i.e.

$$
\begin{aligned}
& C_{\mathrm{PTP}}\left(t, t_{f}, \boldsymbol{p}_{i}, \boldsymbol{p}_{f}\right)=\frac{1}{2} \sum_{\boldsymbol{x}_{f}} e^{i \boldsymbol{p}_{f} \cdot \boldsymbol{x}_{f}} \sum_{m=1}^{3} \sum_{\boldsymbol{x}} e^{-i\left(\boldsymbol{p}_{f}-\boldsymbol{p}_{i}\right) \cdot \boldsymbol{x}}\left\langle P\left(t_{f}, \boldsymbol{x}_{f}\right) T_{0 m}(t, \boldsymbol{x}) P^{\dagger}(0, \mathbf{0})\right\rangle \\
& \quad \times\left\{\left(e^{-i\left(\boldsymbol{\theta}_{f}-\boldsymbol{\theta}_{i}\right)_{m}}-1\right) \delta_{x_{m}, L-1}-\left(e^{i\left(\boldsymbol{\theta}_{f}-\boldsymbol{\theta}_{i}\right)_{m}}-1\right) \delta_{x_{m}, 0}\right\}
\end{aligned}
$$

where $x_{m}$ denotes the $m^{\text {th }}$ component of $\boldsymbol{x}$, while $\boldsymbol{\theta}_{i}, \boldsymbol{\theta}_{f}$ are the twist angles applied to the initial and final pions, respectively.

Our results presented in the following section show that the contribution of the improvement term $c_{\mathrm{V}} C_{\mathrm{PTP}}$ to $C_{3}$ is numerically very small. Moreover, by construction this term vanishes for $Q^{2}=0$, where the form factor is constrained to $f_{\pi \pi}(0)=1$ by symmetry. Given these observations and the fact that no non-perturbative determination of $c_{\mathrm{V}}$ is available for the two-flavour theory we decided to drop the vector current improvement altogether.

\subsection{The pion form factor, the decay constant and the light quark mass}

From now on we set $t_{f}=T / 2$ and drop the corresponding argument in correlation functions. 


\begin{tabular}{cccccc}
\hline \hline$\beta$ & $Z_{\mathrm{A}}$ & 39 & $Z_{\mathrm{P}}$ & 39 & 41 \\
\hline 5.2 & $0.771(6)$ & $0.518(5)$ & -0.0641 & -0.1079 \\
5.3 & $0.778(9)$ & $0.518(5)$ & -0.0506 & -0.0992 \\
5.5 & $0.793(5)$ & $0.518(5)$ & -0.0361 & -0.0848 \\
\hline \hline
\end{tabular}

Table 1: Non-perturbative estimates of renormalisation factors and improvement coefficients, as used in our analysis.

Following ref. [28] one can extract $f_{\pi \pi}\left(Q^{2}\right)$ from ratios of correlation functions such as

$$
\begin{aligned}
& R_{1}\left(t, \boldsymbol{p}_{i}, \boldsymbol{p}_{f}\right)=4 Z_{\mathrm{V}}^{\mathrm{eff}} \sqrt{E\left(\boldsymbol{p}_{i}\right) E\left(\boldsymbol{p}_{f}\right)} \sqrt{\frac{C_{3}\left(t, \boldsymbol{p}_{i}, \boldsymbol{p}_{f}\right) C_{3}\left(t, \boldsymbol{p}_{f}, \boldsymbol{p}_{i}\right)}{C_{P P}\left(T / 2, \boldsymbol{p}_{i}\right) C_{P P}\left(T / 2, \boldsymbol{p}_{f}\right)}}, \\
& R_{2}\left(t, \boldsymbol{p}_{i}, \boldsymbol{p}_{f}\right)=2 \sqrt{E\left(\boldsymbol{p}_{i}\right) E\left(\boldsymbol{p}_{f}\right)} \sqrt{\frac{C_{3}\left(t, \boldsymbol{p}_{i}, \boldsymbol{p}_{f}\right) C_{3}\left(t, \boldsymbol{p}_{f}, \boldsymbol{p}_{i}\right)}{C_{3}\left(t, \boldsymbol{p}_{i}, \boldsymbol{p}_{i}\right) C_{3}\left(t, \boldsymbol{p}_{f}, \boldsymbol{p}_{f}\right)}} .
\end{aligned}
$$

While any multiplicative renormalisation of the vector current cancels in $R_{2}$, the ratio $R_{1}$ is renormalised by the factor $Z_{\mathrm{V}}^{\text {eff }}$. The form factor $f_{\pi \pi}\left(Q^{2}\right)$ can then be determined via

$$
R_{k}\left(t, \boldsymbol{p}_{i}, \boldsymbol{p}_{f}\right)=f_{\pi \pi}\left(Q^{2}\right)\left(E\left(\boldsymbol{p}_{i}\right)+E\left(\boldsymbol{p}_{f}\right)\right), \quad k=1,2,
$$

where it should be kept in mind that this relation is valid for the time component of the vector current and up to corrections induced by excited state contributions. The renormalisation factor $Z_{\mathrm{V}}^{\text {eff }}$ of the vector current has been determined by imposing electric charge conservation, which implies $f_{\pi \pi}(0)=1$, at every value of the lattice spacing. At the non-perturbative level $Z_{\mathrm{V}}^{\mathrm{eff}}$ is obtained by evaluating

$$
Z_{\mathrm{V}}^{\mathrm{eff}}=\frac{C_{P P}(T / 2, \mathbf{0})}{2 C_{3}(t, \mathbf{0}, \mathbf{0})}
$$

We have also computed the renormalised and $\mathrm{O}(a)$ improved current quark mass defined by the PCAC relation

$$
\hat{m} \equiv \frac{Z_{\mathrm{A}}}{Z_{\mathrm{P}}}\left(1+\left[b_{\mathrm{A}}-b_{\mathrm{P}}\right] a m_{q}\right) m_{\mathrm{PCAC}}, \quad m_{\mathrm{PCAC}}=\frac{1}{2} \frac{\left\langle\tilde{\partial}_{0} A_{0}^{I}(x) P^{\dagger}(0)\right\rangle}{\left\langle P(x) P^{\dagger}(0)\right\rangle},
$$

where the bare subtracted quark mass is given by

$$
a m_{q}=\frac{1}{2}\left(\frac{1}{\kappa}-\frac{1}{\kappa_{c}}\right) .
$$

For the critical hopping parameter $\kappa_{c}$ we use the results listed in table 2 . The difference $\left(b_{\mathrm{A}}-b_{\mathrm{P}}\right)$ is taken from [38], and for $Z_{\mathrm{A}}$ and $Z_{\mathrm{P}}$ we use the non-perturbative results from 39], which update the earlier determinations from [40] and [41]. The numerical values of the improvement coefficients and renormalisation factors $Z_{\mathrm{A}}$ and $Z_{\mathrm{P}}$ are listed table 1 .

We use the definition

$$
F_{\pi}^{\text {bare }}=\sqrt{2\left|\mathcal{Z}_{\mathrm{P}}\right|^{2}} \frac{m_{\mathrm{PCAC}}}{m_{\pi}^{2}} .
$$

for the pion decay constant (e.g. 42]). Note, that our normalisation for pseudoscalar decay constants corresponds to a physical value of $F_{\pi}=92.2 \mathrm{MeV} 43$. 


\subsection{Ratios of correlation functions and excited states}

As explained in the previous sections the pion form factor can be determined from the asymptotic form of the ratios 2.14) of two- and three-point correlation functions 2.9) and 2.11), i.e. for large Euclidean time separations between the operator insertions.

One can study the contribution of excited states to the ratios analytically by inserting the spectral decomposition of the two- and three-point functions. We have looked at terms up to and including the first excited state which causes exponentially suppressed deviations from the constant behaviour expected for the ground state (cf. also the study in [10]). The behaviour is the same for $R_{1}$ and $R_{2}$. We control these contributions by choosing $t_{f}$ sufficiently large for all our measurements and by tuning the fit window for every individual result for the ratio such that exponential contaminations are sufficiently decayed.

There is a further, time-independent contamination proportional to $e^{-\Delta t_{f} / 2}$, where $\Delta$ is the energy gap between the ground- and first excited state. We are not able to remove this contribution in our analysis because we do not have data for different choices of $t_{f}$. Under the assumption that $\Delta \approx 2 m_{\pi}$ and for our simulation parameters as summarised table 2 , this contribution is however highly suppressed and it is therefore safe to neglect it.

Note also that due to current conservation at $Q^{2}=0$, which implies $f_{\pi \pi}(0)=1$, the contribution from the first excited state cancels exactly between numerator and denominator. For $Q^{2}>0$ the cancellation is no longer exact, but since it is smoothly connected to vanishing momentum transfer, it is reasonable to assume that excited state contaminations are rather small in the region that is particularly relevant for the determination of the charge radius.

We also note that techniques for a systematic reduction of excited state contaminations have been developed and applied in [44,45]. They will allow for a more precise estimation of residual effects in future calculations of meson form factors.

\section{$3 \quad$ Lattice simulation and results}

\subsection{Simulation parameters}

All our calculations are based on the CLS ${ }^{1}$ ensembles generated with two dynamical flavours of non-perturbatively $\mathrm{O}(a)$ improved Wilson fermions. The simulation parameters, i.e. the bare coupling and the hopping parameter are listed alongside the values of some basic observables in table 2, Gauge ensembles were generated using the DD-HMC [2, 5, 6] and MP-HMC [8] algorithms.

To convert to physical units we use the Sommer scale $r_{0}$ [46], which was recently determined on the CLS ensembles [39, 47]. By computing the kaon decay constant in units of $r_{0}$, i.e. $\left(f_{\mathrm{K}} r_{0}\right)$, taking the continuum limit and combining it with the experimental value of $f_{\mathrm{K}}$, one obtains $r_{0}=0.503(10) \mathrm{fm}[39]$. This value is consistent with the scale setting procedure based on the mass of the $\Omega$-baryon described in [48], provided that the updated results for $r_{0}$ from [39] are used. Hence, the three lattice spacings of the ensembles used here are in the

\footnotetext{
${ }^{1}$ https://twiki.cern.ch/twiki/bin/view/CLS/WebHome
} 


\begin{tabular}{ccccccccc}
\hline \hline & $N_{\text {cfg }}$ & $T \times L^{3}$ & $\beta$ & $r_{0} / a$ & $\kappa_{\text {crit. }}$ & $\kappa_{\text {sea }}$ & $m_{\pi}[\mathrm{MeV}]$ & $m_{\pi} L$ \\
\hline A3 & 132 & $64 \times 32^{3}$ & 5.2 & $6.15(6)$ & $0.136055(4)$ & 0.13580 & 470 & 6.0 \\
A4 & 175 & & & & & 0.13590 & 365 & 4.7 \\
A5 & 108 & & & & & 0.13594 & 310 & 4.0 \\
\hline E4 & 81 & $64 \times 32^{3}$ & 5.3 & $7.26(7)$ & $0.136457(4)$ & 0.13610 & 605 & 6.2 \\
E5 & 119 & & & & & 0.13625 & 450 & 4.6 \\
F6 & 233 & $96 \times 48^{3}$ & & & & 0.13635 & 325 & 5.0 \\
F7 & 250 & & & & & 0.13638 & 280 & 4.3 \\
\hline N3 & 98 & $96 \times 48^{3}$ & 5.5 & $10.00(11)$ & $0.1367749(8)$ & 0.13640 & 630 & 7.6 \\
N4 & 117 & & & & & 0.13650 & 535 & 6.5 \\
N5 & 189 & & & & & 0.13660 & 425 & 5.2 \\
\hline \hline
\end{tabular}

Table 2: Summary of properties of the gauge ensembles. The results for the Sommer scale $r_{0} / a$ and for the critical hopping parameter $\kappa_{\text {crit. }}$ are taken from [39]. We also list approximate values for the pion masses in physical units.

range $0.05 \mathrm{fm}-0.08 \mathrm{fm}$.

We have evaluated all two- and three-point correlation functions using two hits of stochastic $Z_{2} \times Z_{2}$ wall sources $49 \sqrt[52]{ }$. For each configuration subsequent hits were evaluated on two different timeslices which we separated in time by $T / 2$, except for ensemble A5 where we applied hits on four timeslices separated by $T / 4$. The pion mass, the pion decay constant, the PCAC quark mass and the pion vector form factor were evaluated in this way on the ensemble of gauge configurations as summarised in table 4. Our ensembles cover a large range of quark masses and lattice spacings. As a safeguard against large finite-size effects, we have kept $m_{\pi} L \geq 4$ on all ensembles. Our parameter choice thus allows for a comprehensive study of systematic effects, relating to chiral and continuum extrapolations.

As motivated above, we are particularly interested in the region of small momentum transfers. We have therefore tuned the twist angles specifically to achieve a high resolution for small values of $Q^{2}$, using eq. (2.4) and a first rough determination of the pion mass on all ensembles. Twisted boundary conditions were applied in the $x$-direction to the pions in both the initial and final states, whilst projecting on vanishing Fourier momenta, $\boldsymbol{p}_{i}=\boldsymbol{p}_{f}=0$. Table 3 contains the full set of angles used in our simulations. Correlation functions for the pion vector form factor were generated simultaneously with those required for the computation of the $K \rightarrow \pi$ semi-leptonic form factor. For the latter, partially twisted boundary conditions allow for simulating directly at the phenomenologically relevant kinematical point of vanishing momentum transfer 28,53 between the kaon and the pion. The angles $\theta_{1}, \ldots, \theta_{4}$ in table 3 have been tuned such as to realise $Q^{2}=0$ for the $K \rightarrow \pi$ matrix elements. We added one extra twist angle $\theta_{5}$ which was chosen such as to yield a dense set of data points for small momentum transfers.

On each of the ensembles listed in table 2 we have computed the ratios $R_{1}$ and $R_{2}$ in eq. (2.14) for all possible combinations of twists applied to the valence quark being probed by 


\begin{tabular}{ccccccc}
\hline \hline set & $\theta_{0}$ & $\theta_{1}$ & $\theta_{2}$ & $\theta_{3}$ & $\theta_{4}$ & $\theta_{5}$ \\
\hline A4 & 0.0 & \pm 2.2658 & \pm 1.8438 & \pm 1.3582 & \pm 0.7745 & \pm 2.5 \\
A5 & 0.0 & \pm 2.4380 & \pm 2.0281 & \pm 1.5544 & \pm 0.9777 & \pm 2.5 \\
E4 & 0.0 & \pm 1.6799 & \pm 1.2748 & \pm 0.8195 & \pm 0.2969 & \pm 2.5 \\
E5 & 0.0 & \pm 2.1728 & \pm 1.7866 & \pm 1.3476 & \pm 0.8344 & \pm 2.5 \\
F6 & 0.0 & \pm 3.2455 & \pm 2.9371 & \pm 2.6028 & \pm 2.2355 & \pm 1.5 \\
F7 & 0.0 & \pm 3.7892 & \pm 3.5196 & \pm 3.2323 & \pm 2.9231 & \pm 2.0 \\
N3 & 0.0 & \pm 0.7538 & \pm 0.3777 & \pm 0.4935 & \pm 1.0146 & \pm 4.0 \\
N4 & 0.0 & \pm 1.2730 & \pm 0.8936 & \pm 0.4726 & \pm 0.5443 & \pm 3.9 \\
N5 & 0.0 & \pm 1.7513 & \pm 1.3942 & \pm 0.9945 & \pm 0.5311 & \pm 3.2 \\
\hline \hline
\end{tabular}

Table 3: The choice of twist angles applied to the initial and final mesons in the $x$-direction.

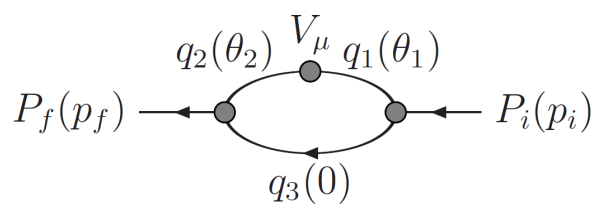

Figure 1: Graphical representation of 3pt-function $C_{3}$ with explanation of twist angles.

the vector current.

\subsection{Data analysis and fitting procedure}

All error estimates are computed by resampling using the bootstrap procedure [54 with 1000 bins. Masses, decay constants, form factor and other quantities have been extracted from correlation functions via suitable fits. While simulation data from a given ensemble are correlated, it is often difficult to obtain sufficiently precise estimates of the full covariance matrix based on a finite set of gauge configurations. As a consequence, numerical instabilities can occur in the least-square minimisations (see for example [55]). We have therefore chosen to quote our main results from uncorrelated fits.

The quantities which we fitted to ChPT often contain input parameters such as renormalisation factors, which have their own intrinsic uncertainties. In order to take the latter into account we have folded them into our analysis via the following procedure: first we generated a pseudo-bootstrap distribution with 1000 bins for the input quantity, whose width was designed such that it reproduced the quoted uncertainty. We checked explicitly that the bootstrap error obtained for the combination of distributions was compatible with the corresponding estimate determined via the usual error propagation.

This procedure was also applied in combinations such as $m_{\pi} r_{0}$, despite the fact that the 


\begin{tabular}{lccccc}
\hline \hline ensemble & $r_{0} m_{\pi}$ & $r_{0} F_{\pi}$ & $r_{0} \hat{m}$ & $Z_{\mathrm{V}}^{\mathrm{eff}}$ & $\left\langle r_{\pi}^{2}\right\rangle / r_{0}^{2}$ \\
\hline \hline A3 & $1.161(12)$ & $0.280(8)$ & $0.090(3)$ & $0.73228(7)$ & $1.14(5)$ \\
A4 & $0.895(11)$ & $0.247(12)$ & $0.052(3)$ & $0.72885(12)$ & $1.20(7)$ \\
A5 & $0.761(11)$ & $0.251(14)$ & $0.040(2)$ & $0.72731(10)$ & $1.48(9)$ \\
\hline E4 & $1.406(16)$ & $0.287(10)$ & $0.128(5)$ & $0.74962(8)$ & $0.98(4)$ \\
E5 & $1.048(13)$ & $0.271(11)$ & $0.078(4)$ & $0.74461(8)$ & $1.18(5)$ \\
F6 & $0.752(8)$ & $0.254(8)$ & $0.041(2)$ & $0.74119(4)$ & $1.37(6)$ \\
F5 & $0.646(7)$ & $0.237(8)$ & $0.029(1)$ & $0.74030(5)$ & $1.61(10)$ \\
\hline N3 & $1.593(18)$ & $0.329(7)$ & $0.188(5)$ & $0.77162(3)$ & $0.90(3)$ \\
N4 & $1.360(16)$ & $0.304(9)$ & $0.139(4)$ & $0.76855(3)$ & $1.04(3)$ \\
N5 & $1.080(13)$ & $0.291(8)$ & $0.091(3)$ & $0.76543(3)$ & $1.17(4)$ \\
\hline \hline
\end{tabular}

Table 4: Results for basic quantities.

determination of $r_{0}$ was mostly performed on the same ensembles. However, since the set of configurations used to compute the pion form factor did not exactly coincide with those used in the calculation of $r_{0}$, we chose to ignore the partial correlation of $r_{0}$ with our data, although this results in a larger overall uncertainty.

\subsubsection{Pion mass and decay constant}

We extracted the pion energy from a cosh-fit to the two-point function $C_{\mathrm{PP}}(t, \boldsymbol{p})$, after checking that the results with our choice of fit-ranges remain unchanged when a three-pion state is included as the first excited state, as suggested in [42]. We obtained the current quark mass $m_{\text {PCAC }}$ from a constant fit to the ratio in eq. (2.17). The bare pion decay constant was then determined from eq. 2.19), using the result for $\mathcal{Z}_{P}$ and $m_{\pi}$ from the above cosh-fit and $m_{\mathrm{PCAC}}$. The fit-results in units of $r_{0}$ are summarised in table 4. They are in agreement with the results obtained in $39{ }^{2}$.

Up to cut-off and exponentially suppressed finite-volume effects, the pion energy obeys the dispersion relation (2.5) where $\boldsymbol{p}$ is the difference of the twist angles applied to the pion's valence quarks $[27,32,56]$ divided by the spatial extent $L$ of the lattice. As an example for how well the continuum dispersion relation is reproduced by our data we show the numerical results on ensemble F6 together with eq. (2.5) in figure 2. Note that for these small momenta the difference between continuum and lattice dispersion relations is negligible. In the remainder of the analysis we use eq. (2.5), i.e. we always determine the kinematics in terms of the pion energy at rest together with the exactly know twist angles.

\footnotetext{
${ }^{2}$ Note that the analysis in $[39]$ was done using different numbers of measurements, source positions and also fitting strategies.
} 


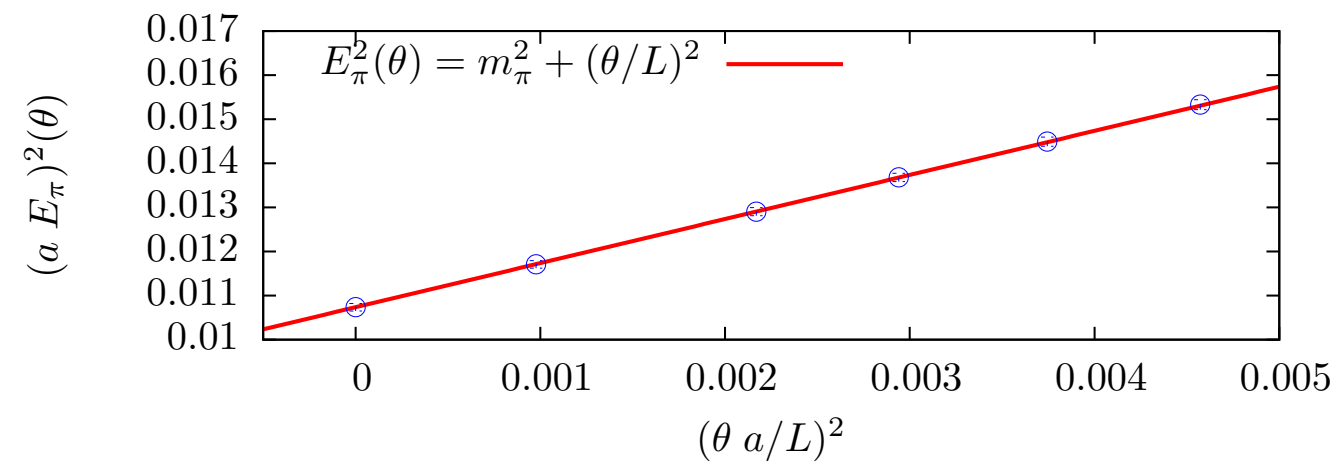

Figure 2: The pion dispersion relation with partially twisted boundary conditions compared to the continuum dispersion relation, eq. (2.5). The vertical axis shows the interval of squared pion momenta up to about $40 \mathrm{MeV}^{2}$.

\subsubsection{Form factor}

As discussed in detail in earlier sections we determine the form factor from the plateau of suitable ratios of two- and three-point functions, $R_{1}$ and $R_{2}$, as defined in eq. 2.14). For each ensemble around 60 kinematical points entered our analysis. We inspected every single plateau by eye for both ratios and then determined the fit range. Some of the plateaus, in particular for larger induced momentum were found to be of unsatisfactory quality. We discarded those ratios from the subsequent analysis. Generally the data for $R_{1}$ was of better quality and we decided to use only results from this ratio in the following. We confirmed however, that using $R_{2}$ instead leads to the same results and conclusions.

Figure 3 shows our results for the form factor for all ensembles. The small inset shows the region of very small $Q^{2}$ which we are concentrating on in this work. In figure 4 we compare our results for the two lightest pions (ensembles F6 and F7) to the experimental measurement, as well as to results of two other lattice collaborations in the region of small $Q^{2}$. The plot illustrates nicely that partially twisted boundary conditions are a powerful method for isolating the low-momentum behaviour of the form factor.

\subsection{Finite volume corrections}

Finite volume effects for the quantities considered here are expected to be suppressed exponentially $\propto e^{-m_{\pi} L}$. In order to keep these effects small we have used only ensembles for which $m_{\pi} L \geq 4$ (cf. table 2) but we have also made the effort to remove residual effects systematically. This was not possible directly using simulation data since we do not have results for different volumes at fixed pion mass at our disposal. Instead we have used predictions of chiral perturbation theory. The corresponding expressions have been derived in [58] for the pion mass and the pion decay constant. Remarkably, the full expressions at NNLO contain only the low-energy constants (LECs) which appear at NLO, which has been referred to as the "elevator-effect" in [58]. For the vector form factor with partially twisted boundary con- 


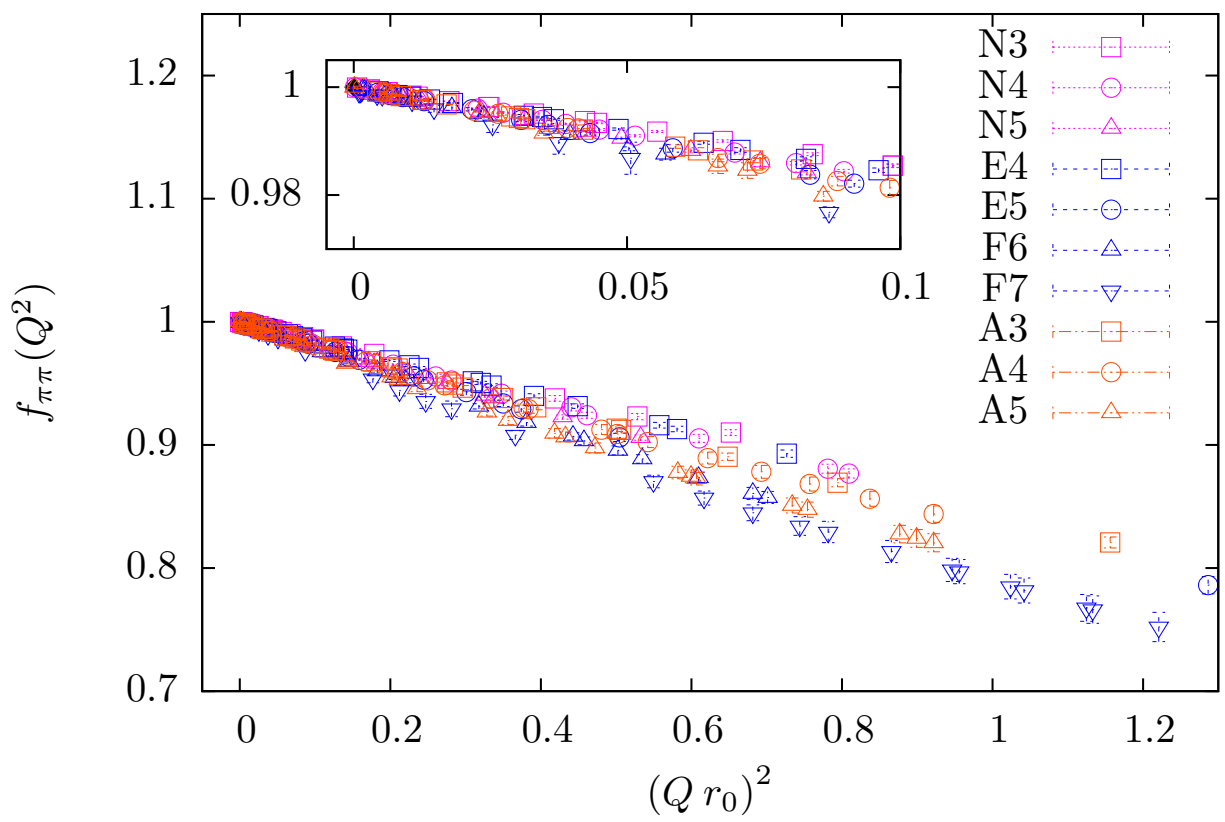

Figure 3: Results for the pion form factor for all ensembles. The inset shows a zoom into the region of small $Q^{2}$.

ditions, finite-volume effects have been computed in NLO chiral perturbation theory [56], for the case that either $\boldsymbol{\theta}_{i}=0$ or $\boldsymbol{\theta}_{f}=0$, and in [59] for the Breit-frame $\boldsymbol{\theta}_{i}=-\boldsymbol{\theta}_{f}$.

In order to evaluate finite-volume effects in ChPT, we initially fixed the relevant LECs in the same way as Colangelo, Dürr and Häfeli [58, who took their values from [60]. To become independent of external input quantities, we performed the following iterative procedure: first, we applied the finite-volume correction based on ChPT and the LECs from [60] as input. The subsequent fits of the chiral behaviour of the lattice data to the expressions of ChPT described in the following section provide us with predictions for the LECs from which the finite-volume shift is re-computed. After repeating this procedure twice no significant change in the output LECs was observed. We note that the estimates after the final iteration are well compatible with the values of 60 .

Despite the fact that the ChPT estimates of finite-volume effects turn out to be numerically small, we apply these corrections to the lattice data prior to any subsequent analysis. In the case of the form factor we restrict ourselves in the following to only those kinematical situations where predictions of finite volume effects are available.

\subsection{Extraction of the charge radius}

The charge radius of the pion is defined as the derivative of the form factor with respect to the momentum transfer at $Q^{2}=0$ (c.f. eq. $(2.2)$ ). In practice, and this also affects the determination from experimental data for the form factor, one fits a model for the $Q^{2}$ dependence to the data (e.g. pole- or polynomial ansatz) and extracts the charge radius in 


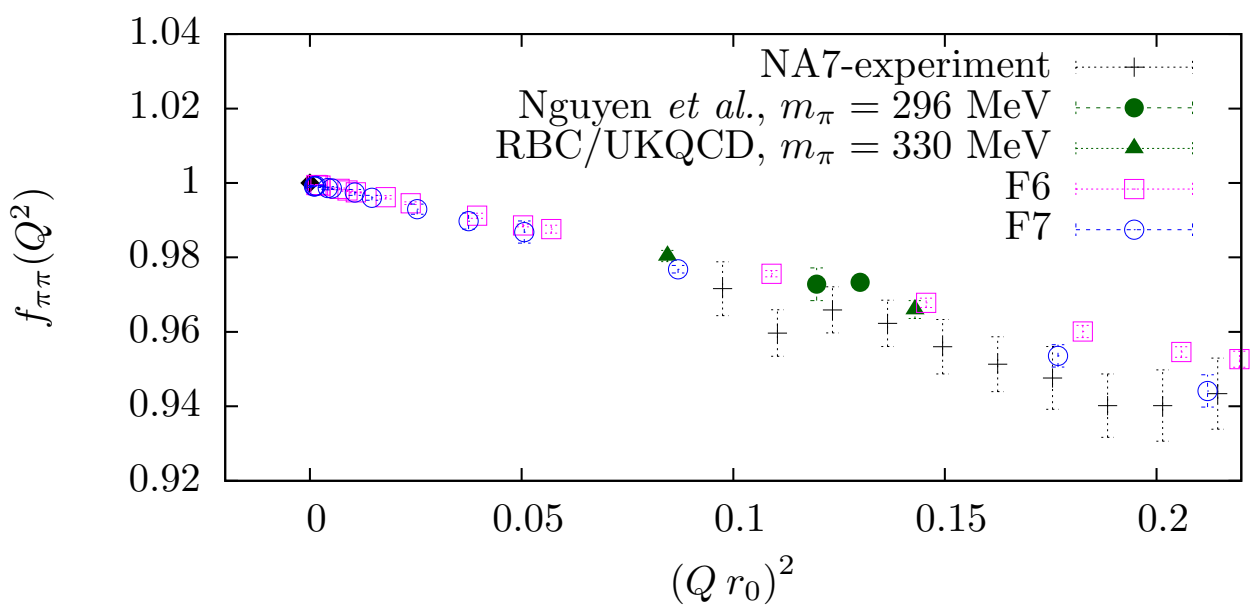

Figure 4: Compilation of results for the pion form factor in dynamical lattice QCD $11,12,14,28$ and as determined from experiment [57].

terms of the slope at the origin. Until recently, data from both experiment and lattice QCD did not cover the region of very low momentum transfer $Q^{2}<0.013 \mathrm{GeV}^{2}$, which is where one would ideally like to extract the slope. Studies of the systematics introduced by the fit-ansatz were therefore very limited.

The high density of data points for $f_{\pi \pi}\left(Q^{2}\right)$ near $Q^{2}=0$ - shown for all ensembles in figure 3 - allows us to constrain the functional form of the form factor very accurately and to reduce any model dependence in the extraction of the charge radius to a minimum. In practice we compare radii as extracted from linear fits in $Q^{2}$, polynomial fits, as well as pole fits of the form

$$
\left.f_{\pi \pi}\left(Q^{2}\right)\right|_{\mathrm{VPD}}=\left(1-\frac{\left\langle r_{\pi}^{2}\right\rangle_{\mathrm{VPD}}}{6} Q^{2}\right)^{-1} .
$$

The latter was already employed in [57] to determine the charge radius from experimental data and is usually used in the determination from lattice data (e.g. $11,12,14,28]$ ).

Figure 5 shows a representative example of the charge radius on ensemble F6, which corresponds to a pion mass of $325 \mathrm{MeV}$. Data points were obtained by fitting the $Q^{2}$-dependence of the form factor to a particular ansatz within an interval $0 \leq Q^{2} \leq Q_{\max }^{2}$. The resulting estimates for the squared charge radius are then plotted versus the value of $Q_{\max }^{2}$ used in the fit. In order to compare results for different mass and lattice spacing, we express all dimensionful quantities in units of $r_{0}$. In the regime of low $Q^{2}$, one observes good agreement between different types of fits. Interestingly, higher orders in $Q^{2}$ turn out to become relevant very early as can be seen from an increasing discrepancy between the linear fit on the one hand, and the polynomial and pole fits on the other.

In the following we will use the result of the fit using a second order polynomial (P2), imposing a cut at $\left(Q_{\max } r_{0}\right)^{2} \approx 0.22$, which in physical units corresponds to about $0.034 \mathrm{GeV}^{2}$. In this range all our ansätze are mutually compatible. We prefer the second-order polynomial over the linear fit, since it yields consistent results over a larger range of $Q_{\max }^{2}$. The results 


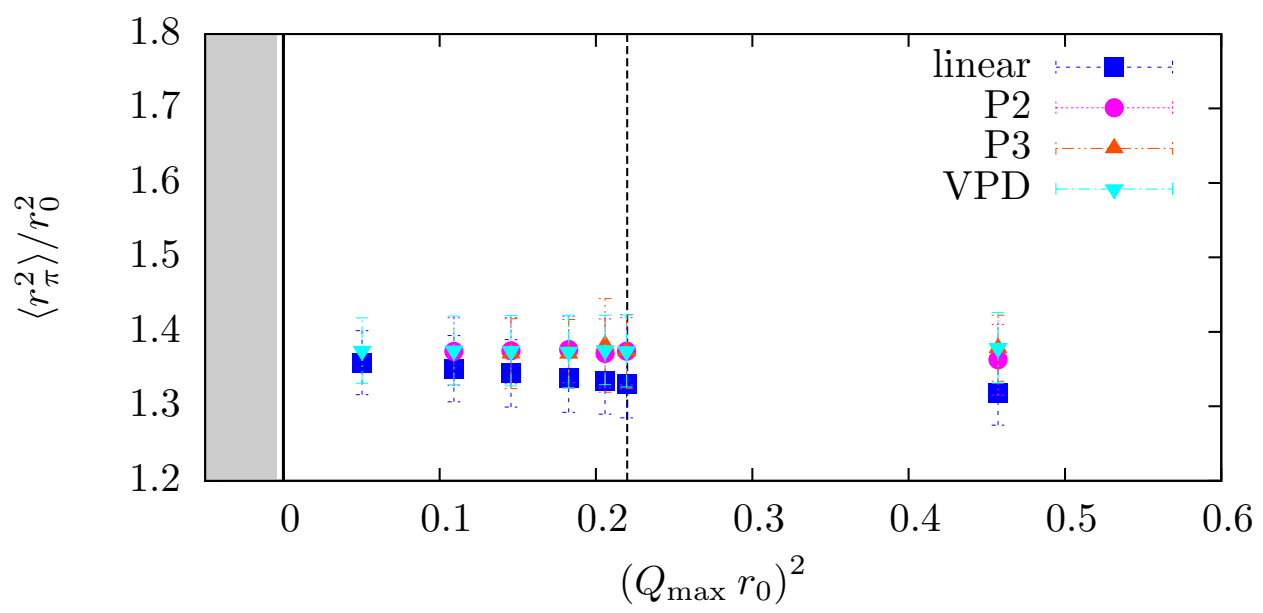

Figure 5: The squared charge radius for a pion mass of about $325 \mathrm{MeV}$ (ensemble F6), plotted versus the maximum value of the $Q^{2}$-interval entering the fit, in units of $r_{0}$. Labels P2 and P3 denote the results of a fit to polynomials of degree two and three, respectively, and the results denoted by VPD are the results of a fit to the form in eq. 3.1.

for $\left\langle r_{\pi}^{2}\right\rangle$ on all ensembles are listed in table 4, and a comparison with results from other collaborations is provided in figure 6 .

Our lattice data suggest that the form factor can be represented very well by a secondorder polynomial up to values of the momentum transfer which have been probed by the NA7-experiment [57].

\section{Chiral and continuum extrapolations}

Table 4 summarises our results for the pion mass, the pion decay constant and the charge radius. We refrain from presenting the abundant numerical data on the form factor itself but will use it for some of the discussions that follow. In this section we present our attempts at parameterising the lattice data and also at extrapolating it to the physical point, i.e. to the physical quark mass and to the continuum and infinite volume limits.

\subsection{Fits guided by chiral perturbation theory}

Chiral perturbation theory [16, 17, 61 provides a comprehensive effective theory framework for describing the low-energy dynamics of QCD. Its predictions for the functional form of the mass-, momentum- and cutoff dependence of low-energy observables are a standard tool in lattice QCD for extrapolating lattice data in parameter space (volume, quark mass, lattice spacing, momentum). For the two flavour theory the expressions for the pion mass, the decay constant and the pion form factor, and consequently also its charge radius, have been computed within this framework at NLO [17,61] and at NNLO [18,62,64]. We summarise the corresponding formulae together with our parameterisation of cutoff effects in appendix A.1 


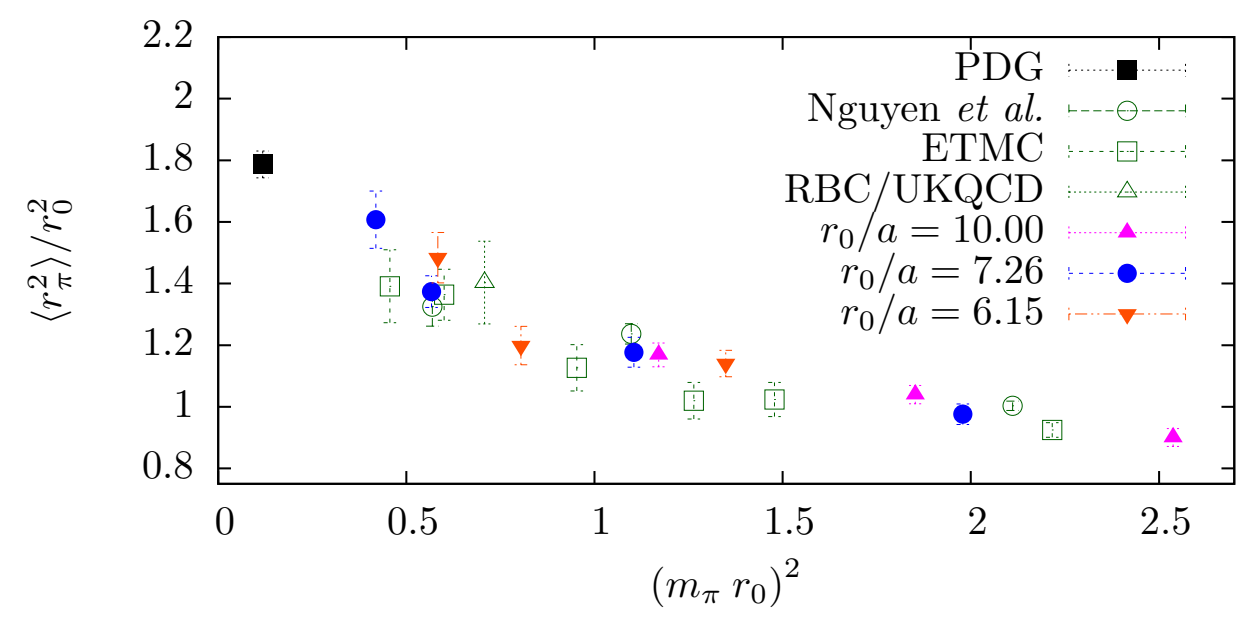

Figure 6: Compilation of results for the pion charge radius in dynamical lattice QCD [1, 12, 14, 28 and the value quoted by the particle data group [43]. Most of the lattice data is extracted from a single pole fit except for the data from RBC/UKQCD and the data from this study, for which we show the quadratic fit based on an identical $Q^{2}$-cut at $\left(Q_{\max } r_{0}\right)^{2} \approx 0.034 \mathrm{GeV}^{2}$.

and A.2. At each order in the expansion new mass- and momentum-independent LECs appear. They are a priori unknown parameters, unconstrained by symmetry, yet they can be determined from lattice QCD data (for a summary of recent results see ref. 9]). Some LECs contribute to the chiral expansion of more than one quantity which can be exploited for correlations and consistency checks. These correlations motivate simultaneous analyses of more than one observable to gain better control over the chiral extrapolations. Here we compare the following fits:

- individual fits to $m_{\pi}^{2}, F_{\pi}, f_{\pi \pi}$ and $\left\langle r_{\pi}^{2}\right\rangle$ at NLO

- joint fits to $\left(m_{\pi}^{2}, F_{\pi}\right)$ at NLO and NNLO

- joint fits to $\left(m_{\pi}^{2}, F_{\pi}, f_{\pi \pi}\right)$ at NLO and NNLO

- joint fits to $\left(m_{\pi}^{2}, F_{\pi},\left\langle r_{\pi}^{2}\right\rangle\right) \quad$ at NLO and NNLO.

Since the chiral series is expected to provide a good representation of QCD only up to a certain low-energy scale we repeat all fits three times including, respectively, all data points for pion masses up to about $430 \mathrm{MeV}, 560 \mathrm{MeV}$ and $630 \mathrm{MeV}$, while monitoring whether the results depend on the choice of the mass cutoff. We have to be less worried about the range of the momentum transfers which enter the fits: The use of partially twisted boundary conditions provides us with many data points well within the realm of chiral perturbation theory. We have extracted the charge radius at very small momentum transfers of up to $\left(r_{0} Q\right)^{2}=0.22$ which in physical units corresponds to about $(190 \mathrm{MeV})^{2}$. For fits to the form factor our choice for the momentum cut is $\left(r_{0} Q\right)^{2}=0.1$ (about $(120 \mathrm{MeV})^{2}$ ) for NLO fits, where the $Q^{2}$-dependence of $f_{\pi \pi}$ is mostly linear (cf. figure 5), and $\left(r_{0} Q\right)^{2}=0.5$ (about $(300 \mathrm{MeV})^{2}$ ) for NNLO fits.

Before discussing the various fits we have performed, it is instructive to recall earlier determinations of low-energy parameters in lattice QCD. The FLAG review [9] quotes global 


\begin{tabular}{|c|c|c|c|c|c|c|c|c|c|}
\hline$m_{\pi}^{\text {cut }}$ & $\chi^{2} / d o f$ & $r_{0} F$ & $r_{0} B$ & $\overline{\ell_{3}}$ & $\bar{\ell}_{4}$ & $\bar{\ell}_{6}$ & $\alpha_{m}$ & $\alpha_{f}$ & $\alpha_{r}$ \\
\hline NLO & $\boldsymbol{F}_{\pi}$ & & & & & & & & \\
\hline all & 0.8 & $0.225(15)$ & & & $4.6(1)$ & & & $-1.3(6)$ & \\
\hline $560 \mathrm{MeV}$ & 0.6 & $0.226(16)$ & & & $4.5(2)$ & & & $-1.2(6)$ & \\
\hline $430 \mathrm{MeV}$ & 0.5 & $0.220(17)$ & & & $4.7(3)$ & & & $-1.2(6)$ & \\
\hline NLO & $\boldsymbol{m}_{\pi}^{2}$ fixed & $F_{\pi}$ & & & & & & & \\
\hline all & 2.1 & & $6.0(4)$ & $2.6(5)$ & & & $-5(1)$ & & \\
\hline $560 \mathrm{MeV}$ & 2.0 & & $6.0(4)$ & $2.3(6)$ & & & $-5(1)$ & & \\
\hline $430 \mathrm{MeV}$ & 1.2 & & $6.2(5)$ & $3.0(8)$ & & & $-4(1)$ & & \\
\hline NLO & $f_{\pi \pi}$ & & & & & & & & \\
\hline all & 1.0 & $0.135(6)$ & & & & $6.7(3)$ & & & $-2(3)$ \\
\hline $560 \mathrm{MeV}$ & 0.9 & $0.140(7)$ & & & & $7.0(4)$ & & & $-2(3)$ \\
\hline $430 \mathrm{MeV}$ & 0.8 & $0.142(8)$ & & & & $7.0(4)$ & & & $-0(3)$ \\
\hline NLO & $\left\langle\boldsymbol{r}_{\boldsymbol{\pi}}^{2}\right\rangle$ & & & & & & & & \\
\hline all & 0.8 & $0.136(6)$ & & & & $6.7(3)$ & & & $-1(3)$ \\
\hline $560 \mathrm{MeV}$ & 0.9 & $0.137(7)$ & & & & $6.8(4)$ & & & $-1(3)$ \\
\hline $430 \mathrm{MeV}$ & 1.2 & $0.137(12)$ & & & & $6.7(7)$ & & & $0(3)$ \\
\hline NLO & $\left\langle\boldsymbol{r}_{\pi}^{2}\right\rangle$ fixe & $\mathrm{d} \boldsymbol{F}_{\pi}$ & & & & & & & \\
\hline all & 6.8 & & & & & $12.0(5)$ & & & $8(3)$ \\
\hline $560 \mathrm{MeV}$ & 6.1 & & & & & $12.7(5)$ & & & $4(3)$ \\
\hline $427 \mathrm{MeV}$ & 4.1 & & & & & $13.6(7)$ & & & $1(3)$ \\
\hline
\end{tabular}

Table 5: Fit results for individual observables based on ChPT at NLO. The coefficients $\alpha_{m}, \alpha_{f}$ and $\alpha_{r}$ parameterise lattice artefacts. For full expressions see appendix A.2.

estimates of $F_{\pi} / F=1.073(15)$ and $\bar{\ell}_{3}=3.2(8)$, while typical results for the LECs $\bar{\ell}_{4}$ and $\bar{\ell}_{6}$ can be summarised as $\bar{\ell}_{4} \approx 4$ and $\bar{\ell}_{6}=12-16$, respectively.

\subsubsection{NLO chiral fits}

All our fit results are compiled in tables 5 and 6. Fits to individual observables using ChPT at NLO are listed in table 5, while table 6 contains results of joint fits to more than one observable, employing both NLO and NNLO expressions. First we note that individual NLO fits to the pion decay constant and mass yield estimates for $F$ as well as the LECs $\bar{\ell}_{3}$ and $\bar{\ell}_{4}$, which are in the same ballpark than those given in the FLAG report (despite the fact that fitting the pion mass with the decay constant fixed at its physical value gives relatively large values of $\chi^{2} /$ dof $)$. In particular, we find $r_{0} F=0.22-0.23$ which, using $r_{0}=0.503 \mathrm{fm}$ from [38], translates into $F=86-90 \mathrm{MeV}$. Given that the PDG quotes the physical pion decay constant as $F_{\pi}^{\text {phys }}=92.2 \mathrm{MeV}$, we find that the ratio $F_{\pi} / F$ determined in this way is completely in line with the global FLAG estimate for this quantity.

However, there is a significant downward shift of about $60 \%$ for $r_{0} F$ when the lattice data for the pion form factor or, alternatively, the charge radius are fitted to the NLO formulae. This very low estimate for $F$ is accompanied by a much smaller value for the LEC $\bar{\ell}_{6}$ compared to the range quoted in the FLAG report. Taken at face value, such fits would suggest chiral corrections as large as $60-70 \%$ between the pseudoscalar decay constant in the chiral limit and at the physical pion mass. Such a scenario contradicts completely the experience gained 


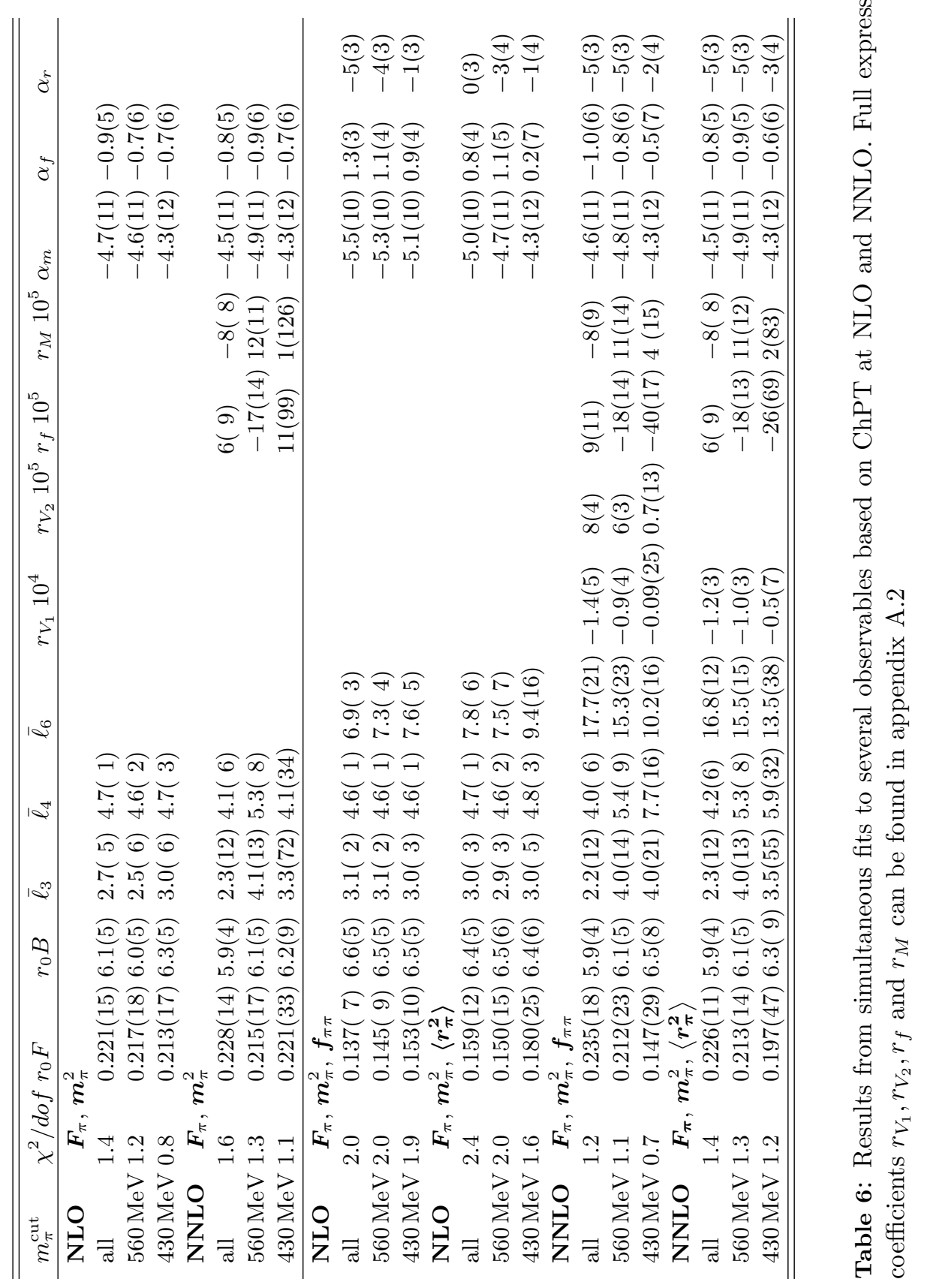


in lattice calculations and effective field theory analyses over many years. Therefore, despite the fact that NLO fits to the form factor and the charge radius have good $\chi^{2} /$ dof, we conclude that the results make no sense. If - on the other hand - one constrains the pion decay constant to its physical value, one finds estimates for $\bar{\ell}_{6}$ which are actually compatible with previous results. However, such fits are not very plausible since their $\chi^{2} /$ dof is unacceptably large.

The failure of the NLO formulae to describe the data for either the form factor or the charge radius in a meaningful way is also manifest in the modelling of cutoff effects of order $a^{2}$. For instance, the value of the coefficient $\alpha_{f}$ determined from a fit to the decay constant $F_{\pi}$ agrees with the result from a joint fit to both $F_{\pi}$ and $m_{\pi}^{2}$ (see the corresponding entries marked "NLO" in tables 5 and 6). When either $f_{\pi \pi}$ or $\left\langle r_{\pi}^{2}\right\rangle$ is included into a joint NLO fit the coefficient $\alpha_{f}$ changes its sign and becomes positive. As can be clearly seen from figure 7 . a positive value of $\alpha_{f}$ is incompatible with the observation that $F_{\pi}$ increases as the lattice spacing is reduced.

To summarise: Chiral Perturbation Theory at NLO fails to produce a consistent description of our lattice data for the entire set of pion observables within the mass range considered in this paper. While individual and joint fits to the pion mass and the pion decay constant lead to a coherent picture, inconsistencies arise when comparing or combining the fits with the data for the form factor or the charge radius.

\subsubsection{NNLO chiral fits}

From the discussion above it is clear that a consistent description of our data may be obtained either by extending the pion mass range to smaller values or by going beyond NLO in ChPT. The NNLO expressions for $F_{\pi}$ and $m_{\pi}$ together contain eight LECs plus two parameters associated with cutoff effects. After including $f_{\pi \pi}$ or $\left\langle r_{\pi}^{2}\right\rangle$ the number of parameters increases to 14 and 13, respectively (cf. A.2). We are thus faced with the problem of having to constrain a large number of parameters with a limited set of data points. It is then not surprising that all our attempts at determining the full set of low-energy parameters were unsuccessful. We note that similar difficulties were encountered by the ETM Collaboration in their two-flavour study of the pion form factor [11]. We therefore decided to stabilise the fits by fixing two of the LECs, $\bar{\ell}_{1}$ and $\bar{\ell}_{2}$, to the values determined from $\pi \pi$-scattering [60], i.e.

$$
\bar{\ell}_{1}=-0.4(5) \quad \text { and } \quad \bar{\ell}_{2}=4.3(1) \text {. }
$$

In the expressions for $m_{\pi}^{2}, F_{\pi}$ and $f_{\pi \pi}$ or $\left\langle r_{\pi}^{2}\right\rangle$ these LECs appear only at NNLO. We checked explicitly that our results do not change significantly when the central values of $\bar{\ell}_{1}$ and $\bar{\ell}_{2}$ are varied by $100 \%$. Moreover, the uncertainties for both LECs are fully included in the analysis, by employing the same procedure described in section 3.2 . Even after reducing the number of free parameters we found that only the joint fits to $\left(F_{\pi}, m_{\pi}^{2}\right)$ on the one hand and either $\left(F_{\pi}, m_{\pi}^{2}, f_{\pi \pi}\right)$ or $\left(F_{\pi}, m_{\pi}^{2},\left\langle r_{\pi}^{2}\right\rangle\right)$ on the other led to stable and consistent results, which are summarised in table 6 . Figures 7 and 8 show the chiral extrapolations with $F_{\pi}, m_{\pi}^{2}$ and $f_{\pi \pi}$ or $\left\langle r_{\pi}^{2}\right\rangle$, respectively, with a mass cut at $560 \mathrm{MeV}$. The statistical uncertainty on the LECs increases noticeably as the upper mass cut is lowered and less data points are allowed to constrain the fit. 

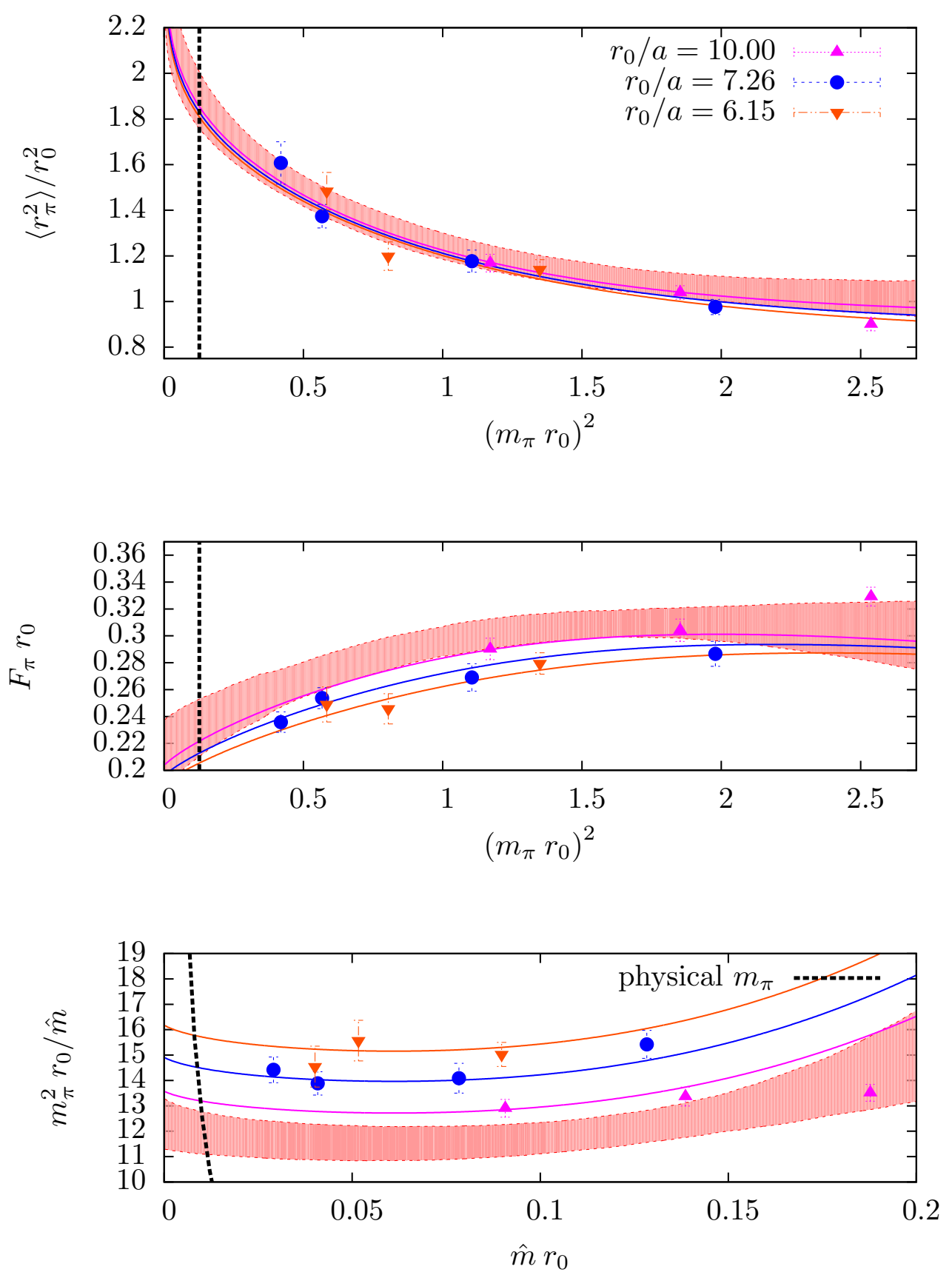

Figure 7: Results from the global fit to the data of $f_{\pi \pi}, F_{\pi}$ and $m_{\pi}$ to ChPT at NNLO with a mass cut at $560 \mathrm{MeV}$. Shown are the chiral extrapolations for $\left\langle r_{\pi}^{2}\right\rangle, F_{\pi}$ and $m_{\pi}$ (from top to bottom). The red band represents the chiral behaviour of the quantity associated with the plot and the different solid lines are the results for the three different lattice spacings. 

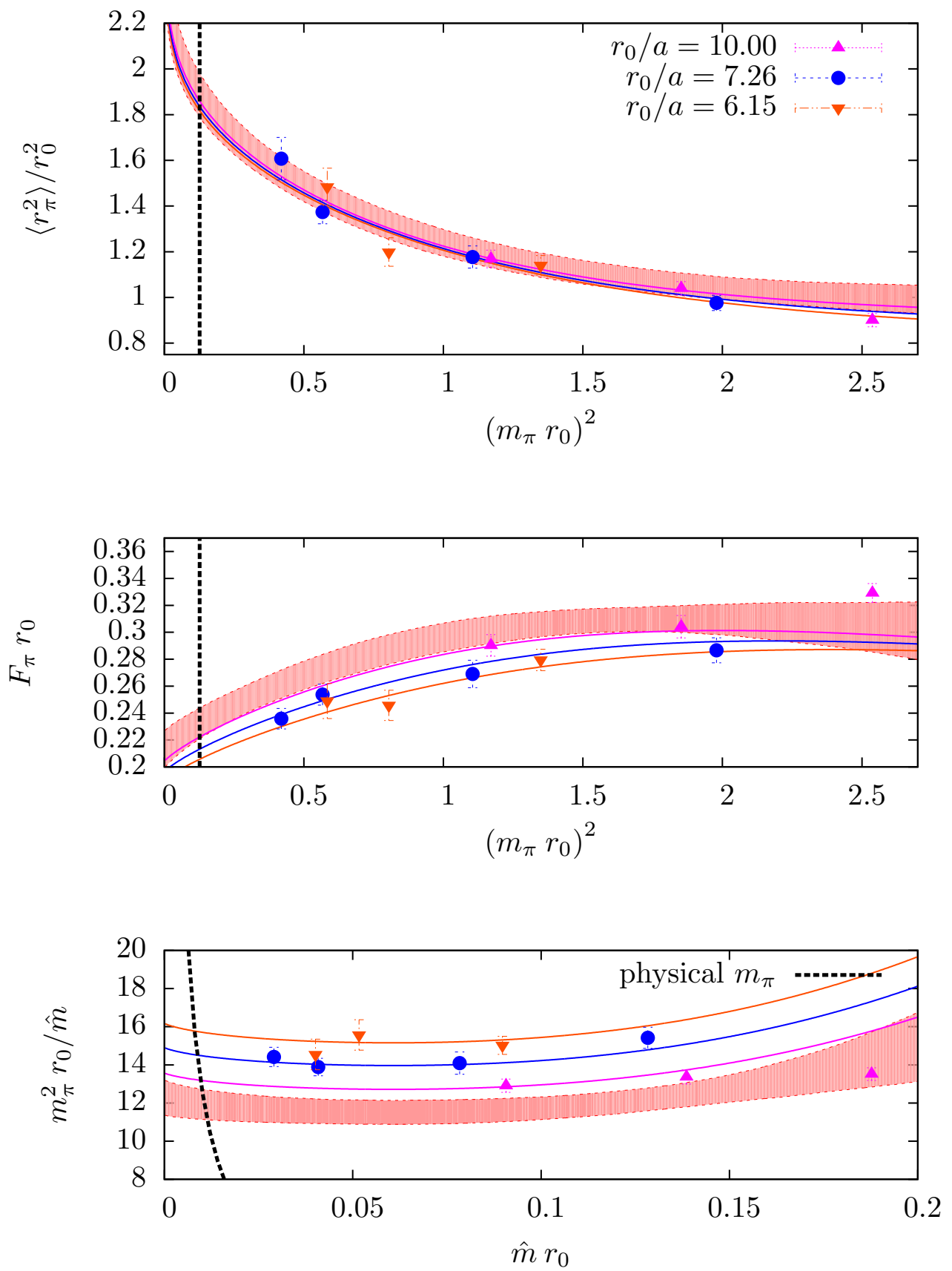

Figure 8: Results from the global fit to the data of $\left\langle r_{\pi}^{2}\right\rangle, F_{\pi}$ and $m_{\pi}$ (from top to bottom) to ChPT at NNLO. The legend is the same as in figure 7 . 


\begin{tabular}{llccccccc}
\hline \hline & & $m_{\pi}^{\text {cut }}$ & $\chi^{2} /$ dof & $r_{0} F$ & $r_{0} B$ & $\bar{\ell}_{3}$ & $\bar{\ell}_{4}$ & $\bar{\ell}_{6}$ \\
\hline $\mathrm{NLO}$ & $F_{\pi}, m_{\pi}^{2}$ & $430 \mathrm{MeV}$ & 0.8 & $0.213(17)$ & $6.3(5)$ & $3.0(6)$ & $4.7(3)$ & \\
$\mathrm{NNLO}$ & $F_{\pi}, m_{\pi}^{2},\left\langle r_{\pi}^{2}\right\rangle$ & $560 \mathrm{MeV}$ & 1.3 & $0.213(14)$ & $6.1(5)$ & $4.0(13)$ & $5.3(8)$ & $15.5(15)$ \\
\hline \hline
\end{tabular}

Table 7: Summary of best fits with NLO and NNLO chiral perturbation theory formulae.

To summarise, the chiral expansion at NNLO provides a consistent description of the data for $F_{\pi}, m_{\pi}^{2}, f_{\pi \pi}$ and $\left\langle r_{\pi}^{2}\right\rangle$. At the current level of precision we do not observe severe inconsistencies like in the case of NLO fits, and thus the results of the NNLO fits appear more trustworthy.

\subsubsection{NLO and NNLO chiral fits: Final results}

In general, all fits based on the NNLO formulae are of reasonable quality in terms of $\chi^{2} /$ dof. However, for a mass cut as low as $430 \mathrm{MeV}$ the fit ceases to be meaningful, as the central values become volatile while the statistical errors increase significantly. We therefore decided to take the simultaneous fit of $F_{\pi}, m_{\pi}^{2}$ and $\left\langle r_{\pi}^{2}\right\rangle$ at NNLO with an upper mass cut of $m_{\pi}^{\text {cut }}=560 \mathrm{MeV}$ as our reference result. Successful fits to NLO expressions can only be achieved by excluding the data for the form factor or charge radius, and we regard the joint fits to $F_{\pi}$ and $m_{\pi}^{2}$ for a mass cut of $430 \mathrm{MeV}$ as our NLO reference results. Table 7 summarises our best fits based on both NLO and NNLO Chiral Pertubation Theory expressions. The LECs extracted from either NLO or NNLO are compatible. Owing to the better statistical accuracy, we take our final results for $F, B, \bar{\ell}_{3}$ and $\bar{\ell}_{4}$ obtained from NLO ChPT as our best overall estimates. The LEC $\bar{\ell}_{6}$ is extracted from the global NNLO fit.

\subsection{Fits guided by polynomial models}

All fits to the lattice data carried out so far took advantage of a firm theoretical prediction, based on chiral dynamics, for the dependence of several observables on the pion mass and momentum transfer, in terms of a common set of low-energy parameters. Nevertheless, it is interesting to study the ability of simple fit ansätze, for which the expansion is not constrained by symmetries, to describe the data. One such model is a simple polynomial in the square of the pion mass. Clearly, in the absence of an underlying dynamical theory which relates different observables, a global fit to, say, $\left\langle r_{\pi}^{2}\right\rangle, F_{\pi}$ and $m_{\pi}^{2}$ makes little sense. Here we only consider $\left\langle r_{\pi}^{2}\right\rangle$ using the ansatz

$$
\frac{\left\langle r_{\pi}^{2}\right\rangle}{r_{0}^{2}}=b_{0}+b_{a}\left(\frac{a}{r_{0}}\right)^{2}+b_{1}\left(r_{0} m_{\pi}\right)^{2}+b_{2}\left(r_{0} m_{\pi}\right)^{4}+\ldots
$$

where we have scaled all dimensionful quantities in units of $r_{0}$. The results are summarised in table 8. As indicated by the value of $\chi^{2} /$ dof, the fits are of reasonable quality, despite the fact that, contrary to the ansätze used in the previous sections, no chiral logarithms are taken into account. We do, however, find an unsatisfactory dependence of the extrapolated 


\begin{tabular}{l|cccccc}
\hline \hline Order & $m_{\pi}^{\text {cut }}$ & $b_{0}$ & $b_{a}$ & $b_{1}$ & $b_{2}$ & $\chi^{2} /$ dof \\
\hline \hline$m_{\pi}^{4}$ & all & $1.68(9)$ & $-1(3)$ & $-0.50(10)$ & $0.08(3)$ & 1.4 \\
& $560 \mathrm{MeV}$ & $1.77(13)$ & $-2(3)$ & $-0.67(19)$ & $0.15(7)$ & 1.4 \\
& $430 \mathrm{MeV}$ & $2.20(27)$ & $-2(4)$ & $-1.70(57)$ & $0.72(32)$ & 1.0 \\
\hline$m_{\pi}^{6}$ & all & $1.92(19)$ & $-1(3)$ & $-1.1(4)$ & $0.5(3)$ & 1.1 \\
& $560 \mathrm{MeV}$ & $2.44(32)$ & $-0(3)$ & $-2.8(10)$ & $2.1(9)$ & 0.6 \\
& $430 \mathrm{MeV}$ & $3.08(87)$ & $1(5)$ & $-5.3(35)$ & $5.0(41)$ & 0.9 \\
\hline \hline
\end{tabular}

Table 8: Results for the coefficients of the naive polynomial model for $\left\langle r_{\pi}^{2}\right\rangle$ as defined via eq. 4.2.

charge radius, when the mass cut is lowered to $430 \mathrm{MeV}$. This may be ascribed to the stronger sensitivity of polynomial fits to fluctuations in the data near the physical pion mass. We note that an additional term $b_{3}$ in the fit including a $m_{\pi}^{6}$-term is not properly determined by our data set. Figure 9 illustrates the extrapolations via polynomials for the cases of a polynomial to $\mathcal{O}\left(m_{\pi}^{4}\right)$ with all data points and also for a mass cut imposed at $560 \mathrm{MeV}$. As can be seen, both extrapolations describe the data well and yield compatible results for the whole range of pion masses.

\subsection{Results at the physical point}

We can now use the fit results from the previous section to determine the pion charge radius and decay constant in the continuum limit and at the physical pion mass. The latter is understood as the mass of the charged pion in QCD, $\left(m_{\pi^{+}}\right)^{\mathrm{QCD}}$, i.e. with the electromagnetic contributions subtracted. Following the discussion in section 3.1 of the FLAG report [9], we find that $\left(m_{\pi^{+}}\right)^{\mathrm{QCD}}$ is, to a good approximation, given by the physical mass of the neutral pion, i.e.

$$
\left(m_{\pi^{+}}\right)^{\mathrm{QCD}} \simeq m_{\pi^{0}}=135 \mathrm{MeV} .
$$

The combined chiral and continuum fits yield the values of the fit parameters in the continuum limit. Furthermore, we have corrected our input data for finite-size effects, as described in section 3.3 .

The results are summarised in table 9, where we have only included those fits, for which the ratio $F_{\pi} / F$ does not deviate from unity by more than $10 \%$ and which also have acceptable values of $\chi^{2} /$ dof. We find that the estimates for the physical pion decay constant and charge radius show practically no variation outside the quoted statistical errors, with the possible exception of the charge radius extracted from polynomial fits. As the mass cut is decreased to $430 \mathrm{MeV}$, the statistical accuracy of NNLO fits deteriorates.

In the following we discuss the various sources of systematic error. We note that the uncertainties of input parameters, such as renormalisation factors or the estimates for the LECs $\bar{\ell}_{1}$ and $\bar{\ell}_{2}$ were folded into the analysis.

Cutoff effects - Our data are compatible with ansätze assuming a linear dependence on $a^{2}$, and at the current level of precision we are not sensitive to higher-order lattice artefacts. In 


\begin{tabular}{lllll}
\hline \hline fit & & $m_{\pi}^{\text {cut }}$ & $F_{\pi} r_{0}$ & $\left\langle r_{\pi}^{2}\right\rangle / r_{0}^{2}$ \\
\hline $\mathrm{NLO}$ & $F_{\pi}$ & all & $0.241(14)$ & \\
$\mathrm{NLO}$ & $F_{\pi}, m_{\pi}$ & & $0.237(14)$ & \\
$\mathrm{NNLO}$ & $F_{\pi}, m_{\pi}$ & & $0.241(13)$ & \\
$\mathrm{NNLO}$ & $F_{\pi}, m_{\pi},\left\langle r_{\pi}^{2}\right\rangle$ & & $0.240(11)$ & $1.85(9)$ \\
$\mathrm{NNLO}$ & $F_{\pi}, m_{\pi}, f_{\pi \pi}$ & $0.248(17)$ & $1.82(10)$ \\
poly & $\left\langle r_{\pi}^{2}\right\rangle, \mathcal{O}\left(m_{\pi}^{4}\right)$ & & & $1.62(8)$ \\
\hline $\mathrm{NLO}$ & $F_{\pi}$ & $560 \mathrm{MeV}$ & $0.241(15)$ & \\
$\mathrm{NLO}$ & $F_{\pi}, m_{\pi}$ & & $0.233(16)$ & \\
$\mathrm{NNLO}$ & $F_{\pi}, m_{\pi}$ & & $0.233(15)$ & \\
$\mathrm{NNLO}$ & $F_{\pi}, m_{\pi},\left\langle r_{\pi}^{2}\right\rangle$ & & $0.231(12)$ & $1.90(10)$ \\
$\mathrm{NNLO}$ & $F_{\pi}, m_{\pi}, f_{\pi \pi}$ & & $0.231(20)$ & $1.90(12)$ \\
poly & $\left\langle r_{\pi}^{2}\right\rangle, \mathcal{O}\left(m_{\pi}^{4}\right)$ & & & $1.70(11)$ \\
\hline $\mathrm{NLO}$ & $F_{\pi}$ & \multirow{2}{*}{$430 \mathrm{MeV}$} & $0.236(15)$ & \\
$\mathrm{NLO}$ & $F_{\pi}, m_{\pi}$ & & $0.230(16)$ & \\
$\mathrm{NNLO}$ & $F_{\pi}, m_{\pi}$ & & $0.235(22)$ & \\
$\mathrm{NNLO}$ & $F_{\pi}, m_{\pi},\left\langle r_{\pi}^{2}\right\rangle$ & & $0.219(32)$ & $1.89(29)$ \\
$\mathrm{NNLO}$ & $F_{\pi}, m_{\pi}, f_{\pi \pi}$ & $0.186(20)$ & $2.30(34)$ \\
poly & $\left\langle r_{\pi}^{2}\right\rangle, \mathcal{O}\left(m_{\pi}^{4}\right)$ & & $2.00(21)$ \\
\hline $\mathrm{PDG}$ & & $0.235(4)$ & $1.79(5)$ \\
\hline \hline
\end{tabular}

Table 9: Results for the pion decay constant and charge radius at the physical point in units of $r_{0}$. The PDG values have been converted using $r_{0}=0.503(10) \mathrm{fm}$ [39]. 


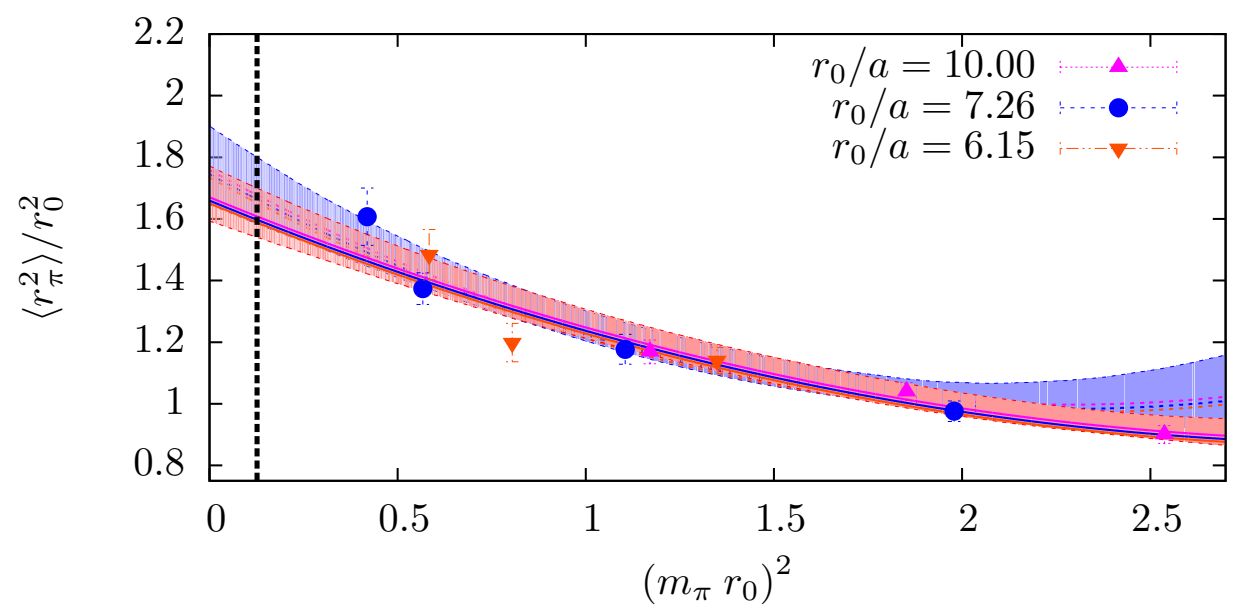

Figure 9: Result for the fit to the form in eq. 4.2 to order $m_{\pi}^{4}$, including lattice artefacts. The red band is the result for the fit in the continuum to all data points and the blue band is the result for the fit with a mass cut of $560 \mathrm{MeV}$. The different colours emphasise different lattice spacings. The solid and dashed lines are the fit result for the different lattice spacings and all data points and the mass cut at $560 \mathrm{MeV}$, respectively. For the results at the different lattice spacings the error bars are left out for the purpose of visibility.

order to estimate the size of residual discretisation errors of order $a^{4}$ we make the following exercise. First, we note that the global fit to $m_{\pi}^{2}, F_{\pi}$ and $\left\langle r_{\pi}^{2}\right\rangle$ with $m_{\pi}^{\text {cut }}=560 \mathrm{MeV}$ suggests that the corrections of order $a^{2}$, estimated as the difference between the continuum limit and the coarsest lattice spacing, amount to $10 \%$ for the pion decay constant and $7 \%$ for the charge radius. This is in the same ballpark than the crude estimate of $\mathrm{O}\left(a^{2}\right)$ lattice artefacts of $\left(\Lambda_{\mathrm{QCD}} a\right)^{2} \approx 4 \%$ (where we have used $\left.\Lambda_{\mathrm{QCD}} \approx 500 \mathrm{MeV}\right)$. By the same argument one can give a rough estimate of $\mathrm{O}\left(a^{4}\right)$ cutoff effects, which then amounts to $0.2 \%$. Compared to the typical statistical accuracy this error is negligible.

Finite size effects - All our ensembles satisfy $m_{\pi} L>4$, which has often been considered sufficient to guarantee small effects due to the finiteness of the box size. In addition, we have corrected for finite-volume effects on the pion mass and decay constant using ChPT at NNLO and ChPT at NLO for the form factor and charge radius. We believe that the residual finite volume effects are negligible.

Chiral extrapolation - The only globally consistent extrapolation of the lattice data was achieved using NNLO chiral perturbation theory, while NLO turned out to be sufficient when fitting only $F_{\pi}$ and $m_{\pi}$. We estimated the residual uncertainty due to chiral extrapolation from the spread of results obtained considering different mass cuts. For the results covered by the NLO extrapolation (i.e. $F, F_{\pi}, \bar{\ell}_{3}, \bar{\ell}_{4}$ and $B$ ) we used the difference between $m_{\pi}^{\text {cut }}=430 \mathrm{MeV}$ and $560 \mathrm{MeV}$ as the symmetric systematic error due to the chiral extrapolation. For $\left\langle r_{\pi}^{2}\right\rangle$ and $\bar{\ell}_{6}$ determined via the NNLO fit we use the spread between the central value between the fit over all data and the one with an upper mass cutoff of $560 \mathrm{MeV}$. 
Scale setting - The Sommer scale [46] was used to combine data obtained at different values of the lattice spacing and to convert to physical units. The absolute physical scale was set by the kaon leptonic decay constant [39], and the result for the scale is fully compatible with the independent determination using the mass of the Omega baryon 48. The associated errors were folded into the analysis during the resampling (c.f. section 3.2).

Critical slowing down - It has been known for some time that simulations of lattice QCD suffer from critical slowing down, which rapidly accelerates when approaching the continuum limit 65 67]. While promising ideas for reducing the severity of the problem in future simulations have by now been developed [68,69], we cannot exclude the possibility that the results of this paper which are based on the ensembles at our finest lattice spacing (N3, N4 and N5) are affected. One consequence for the data analysis, the underestimation of autocorrelations and hence the underestimation of statistical errors, was studied in [67] where a procedure for estimating this effect has been devised. The limited number of measurements in the present work, however, do not allow for a similar treatment of the observables considered here. In this situation we have mimicked the effect of an underestimation of the statistical error in all results generated from ensembles $\mathrm{N} 3, \mathrm{~N} 4$ and $\mathrm{N} 5$, by inflating the statistical error by a factor of two prior to all subsequent analysis. The size of the inflated error is suggested by the findings of [39, 67]. In general, the central values of the final results hardly change, and only the statistical error increases slightly. In the following we adopt this statistical error when quoting the final results but keep the central values from the analysis without the inflated error.

We now summarise our final results. Since the combined NLO fits to the pion decay constant and the pion mass are of good quality and statistical accuracy, we use it to quote final results for the bulk of the fitted low-energy parameters, imposing a mass cut of $430 \mathrm{MeV}$ :

$$
\begin{aligned}
& F_{\pi}=90(8)(2) \mathrm{MeV}, \quad F=84(8)(2) \mathrm{MeV}, \\
& F_{\pi} / F=1.080(16)(6), \quad B=2.5(3)(1) \mathrm{GeV} \text {, } \\
& \bar{\ell}_{3}=3.0(7)(5), \quad \bar{\ell}_{4}=4.7(4)(1) \text {. }
\end{aligned}
$$

Note that the low energy constant $B$ depends on the renormalisation scheme. Here we quote the result in the $\overline{\mathrm{MS}}$-scheme at $\mu=2 \mathrm{GeV}$. Since the product of $B$ and the current (PCAC) quark mass is scale and scheme independent, the LEC $B$ in the $\overline{\mathrm{MS}}$-scheme is obtained after dividing by the renormalisation factor of the quark mass. For our chosen discretisation, this factor $(0.968(20))$ is easily determined using the results of refs. 39,41.

For the pion charge radius and the LEC $\bar{\ell}_{6}$ we take the results from the combined NNLO-fit to the pion decay constant, the pion mass and the pion charge radius,

$$
\begin{aligned}
& \left\langle r_{\pi}^{2}\right\rangle=0.481(34)(13) \mathrm{fm}^{2} \\
& \bar{\ell}_{6}=15.5(1.7)(1.3) .
\end{aligned}
$$

In each case the first error is statistical and the second one is due to the chiral extrapolation, as explained above. Other systematic effects are much smaller and have therefore not been specified.

We end this section with the observation that our results for $F_{\pi} / F, \bar{\ell}_{3}$ and $\bar{\ell}_{4}$ are in very good agreement with the values listed in section 4 of the FLAG report [9]. Furthermore, we 


\begin{tabular}{lcc|l|l|l|l}
\hline \hline & ref & $N_{f}$ & chiral extrapolation & $\left\langle r_{\pi}^{2}\right\rangle$ extr. & $\left\langle r_{\pi}^{2}\right\rangle / r_{0}^{2}$ & $\left\langle r_{\pi}^{2}\right\rangle\left[\mathrm{fm}^{2}\right]$ \\
\hline \hline this study & $\overline{2}$ & 2 & NNLO ChPT $\left(\left\langle r_{\pi}^{2}\right\rangle, F_{\pi}, m_{\pi}\right)$ & poly. $\mathcal{O}\left(q^{4}\right)$ & $1.90(11)$ & $0.481(33)(13)$ \\
\hline QCDSF & $\overline{10}$ & 2 & poly. for $M_{\text {pole }}$ & pole & $2.027(89)$ & $0.442(19)$ \\
ETMC & $\overline{11}$ & 2 & NNLO ChPT $\left(\left\langle r_{\pi}^{2}\right\rangle, F_{\pi}, m_{\pi}\right)$ & pole & & $0.456(30)(24)$ \\
JLQCD/TWQCD & $\overline{13}$ & 2 & NNLO ChPT $\left(\left\langle r_{\pi}^{2}\right\rangle, F_{\pi}, m_{\pi}\right)$ & pole & $1.703(96)$ & $0.409(23)(37)$ \\
RBC/UKQCD & $\overline{12}$ & $2+1$ & NLO ChPT $\left(f_{\pi \pi}\left(Q^{2}\right)\right)$ & NLO & & $0.418(31)$ \\
Nguyen et al. & $\overline{14}$ & $2+1$ & NNLO ChPT $\left(\left\langle r_{\pi}^{2}\right\rangle, F_{\pi}, m_{\pi}\right)$ & pole & & $0.441(46)$ \\
\hline PDG & $\overline{43}$ & - & - & gl. av. & & $0.452(11)$ \\
Amendolia et al. & $\mathbf{5 7}$ & - & - & pole & & $0.439(8)$ \\
BCT & $\mathbf{1 8}$ & - & - & NNLO & & $0.437(16)$ \\
\hline \hline
\end{tabular}

Table 10: Compilation of results for the charge radius at the physical point from lattice QCD $10-14$ 28, including this study, experiment [43,57] and ChPT to NNLO for the experimental data [18]. See also scatter plot in figure 10

can use the well-known Gell-Mann-Oakes-Renner (GMOR) relation 70

$$
\Sigma=F^{2} B
$$

which relates the LECs $B$ and $F$ to the quark condensate $\Sigma$. Our result for the condensate in the $\overline{\mathrm{MS}}$-scheme at a renormalisation scale of $\mu=2 \mathrm{GeV}$ is

$$
\Sigma^{1 / 3}=261(13)(1) \mathrm{MeV},
$$

which is also in good agreement with the results listed in 9 .

\section{Conclusions and outlook}

We have presented the first determination of the iso-vector electromagnetic form factor which does not rely on any particular model for the $Q^{2}$-dependence of the form factor. Our study in two-flavour QCD has full control over the main systematic uncertainties, except isospin breaking effects. A crucial ingredient was the extensive use of partially twisted boundary conditions, which allowed us to achieve a high resolution of data points for the form factor close to $Q^{2}=0$, thereby enabling a model-independent determination of the charge radius.

Our data for the pion mass, decay constant, form factor and charge radius were then subjected to extensive fits to ChPT at NLO and NNLO, augmented by terms which parameterise leading lattice artefacts. While the NLO expressions failed to produce a consistent description of all observables, individual or joint fits to the data of the decay constant and mass lead to a coherent picture. This indicates a problem with the effective theory description of the form factor at NLO at least for the range of quark masses conisdered here. At the level of statistical precision of the lattice data achieved here the NNLO expressions on the other hand allow for a fully consistent description of all observables. The proliferation of free parameters at NNLO could be dealt with by fixing two LECs, $\bar{\ell}_{1}$ and $\bar{\ell}_{2}$, from $\pi \pi$ scattering.

The ability of ChPT to describe lattice data generally depends on the mass range considered in simulations. We note that our conclusions have been reached for pion masses between 280 


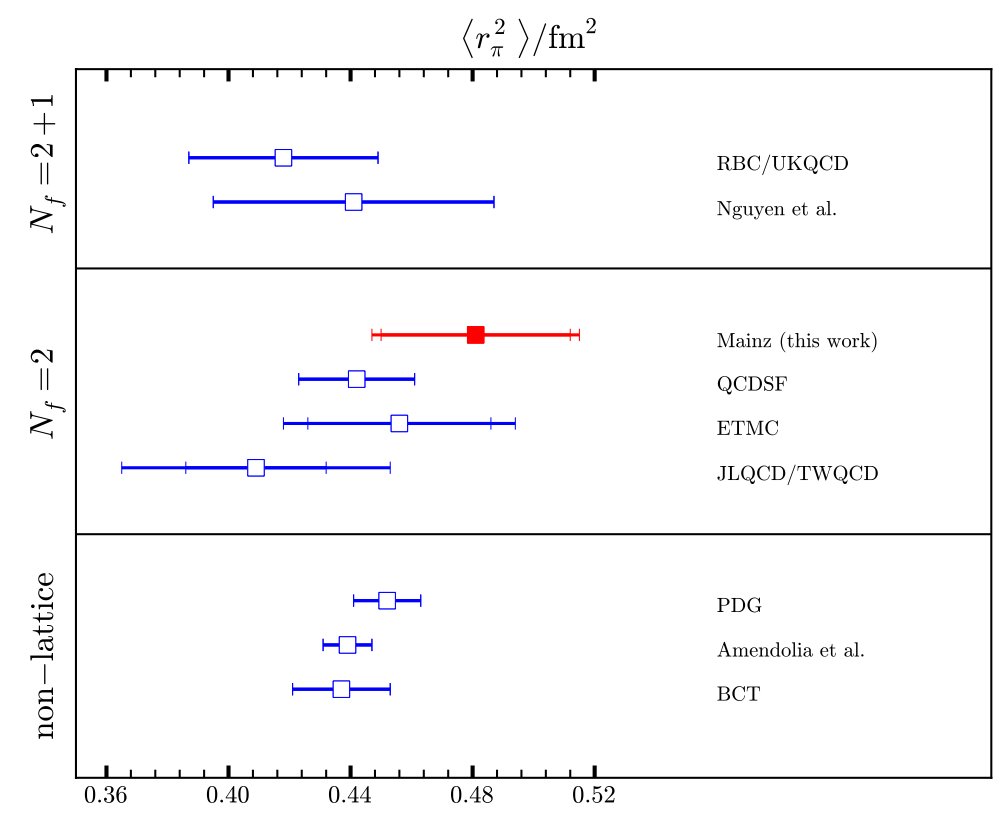

Figure 10: Comparison of results for the charge radius, cf. table 10.

and $540 \mathrm{MeV}$. It will be interesting to study whether ChPT at NLO can be successfully fitted to the data including the form factor or the charge radius, when data at or near the physical pion mass become available.

In table 10 and figure 10 we compare our result for the charge radius to those from other lattice simulations, experimental determinations, as well as results from a ChPT description of experimental data. Despite the relatively good agreement between the various lattice estimates and the value quoted by the PDG [43], one observes a certain spread among the lattice results, with the result of this study at the upper end. In some cases the differences can be traced to the scale setting procedure $3^{3}$ It is then clear that further efforts in lattice QCD are required to pin down the pion charge radius with better overall accuracy. The need for additional simulations - preferably at the physical pion mass - is further highlighted by the difficulties which we encountered in obtaining a consistent ChPT description of the data for the form factor and charge radius.

Acknowledgements: We are grateful to our colleagues within the CLS project for sharing gauge ensembles. Calculations of correlation functions were performed on the dedicated QCD platform "Wilson" at the Institute for Nuclear Physics, University of Mainz and on the QCD HPC "thqcd2" cluster at CERN. We thank Dalibor Djukanovic for technical support. This work was supported by DFG (SFB443 and SFB TR55 "Hadron Physics from Lattice QCD") and the Research Center EMG funded by Forschungsinitiative Rheinland-Pfalz. The research

\footnotetext{
${ }^{3}$ For instance, the QCDSF Collaboration quotes $r_{0}=0.467 \mathrm{fm}$, while we take the more recent determination of ref. 39, i.e. $r_{0}=0.503(10) \mathrm{fm}$
} 
leading to these results has also received funding from the European Research Council under the European Community's Seventh Framework Programme (FP7/2007-2013) ERC grant agreement No 279757.

\section{A Appendix}

\section{A.1 Chiral perturbation theory to NNLO}

For the purpose of performing a global fit to the data of $m_{\pi}, F_{\pi}$ and $f_{\pi \pi}\left(Q^{2}\right)$ (or $\left\langle r_{\pi}^{2}\right\rangle$ alternatively) we first review the formulae of chiral perturbation theory to NNLO as given in [18. For convenience we adopt their notation and define the quantities

$$
\begin{array}{rlrl}
x_{2} & \equiv \frac{m_{\pi}^{2}}{F_{\pi}^{2}} & m_{0} & \equiv 2 B \hat{m} \\
N & \equiv 16 \pi^{2} & L & \equiv \frac{1}{N} \ln \left(\frac{m_{\pi}^{2}}{\mu^{2}}\right)
\end{array}
$$

Here $\mu$ is the renormalisation scale which we set to $\mu=m_{\rho}^{p h y s}=0.77 \mathrm{GeV}[43]$ and $\hat{m}$ is the renormalised bare quark mass. Note that a different renormalisation of $\hat{m}$ is absorbed in a different renormalisation of the LEC $B$. The LECs that are scale-independent are denoted as $\bar{\ell}_{i}$ and are given in terms of the renormalised LECs at the physical pion mass. For these we define the related scale-dependent quantities

$$
\ell_{i}^{r} \equiv \frac{\gamma_{i}}{2 N}\left(\bar{\ell}_{i}+\left.N L\right|_{m_{\pi}^{\text {phys }}}\right) \quad \text { and } \quad k_{i} \equiv\left(4 \ell_{i}^{r}-\gamma_{i} L\right) L
$$

which appear in the formulae. Here $\gamma_{i}$ are the anomalous dimensions, given by

$$
\gamma_{1}=1 / 3, \quad \gamma_{2}=2 / 3, \quad \gamma_{3}=-1 / 2, \quad \gamma_{4}=2, \quad \gamma_{6}=-1 / 3,
$$

and $\left.L\right|_{m_{\pi}^{\text {phys }}}$ denotes the chiral logarithm with the physical pion mass in the numerator. We further define the functions

$$
\begin{aligned}
J\left(\bar{q}^{2}\right) & \equiv \frac{\sqrt{z}}{N} \ln \left(\frac{\sqrt{z}-1}{\sqrt{z}+1}\right)+\frac{2}{N} \\
K_{1}\left(\bar{q}^{2}\right) & \equiv z h^{2}\left(\bar{q}^{2}\right) \\
K_{2}\left(\bar{q}^{2}\right) & \equiv z^{2} h^{2}\left(\bar{q}^{2}\right)-\frac{4}{N^{2}} \\
K_{3}\left(\bar{q}^{2}\right) & \equiv \frac{N z}{\bar{q}^{2}} h^{3}\left(\bar{q}^{2}\right)+\frac{\pi^{2} h\left(\bar{q}^{2}\right)}{N \bar{q}^{2}}-\frac{\pi^{2}}{2 N^{2}} \\
K_{4}\left(\bar{q}^{2}\right) & \equiv \frac{1}{\bar{q}^{2} z}\left(\frac{1}{2} K_{1}\left(\bar{q}^{2}\right)+\frac{1}{3} K_{3}\left(\bar{q}^{2}\right)+\frac{1}{N} J\left(\bar{q}^{2}\right)+\frac{\left(\pi^{2}-6\right) \bar{q}^{2}}{12 N^{2}}\right),
\end{aligned}
$$

with

$$
z \equiv 1-\frac{4}{\bar{q}^{2}} \quad \text { and } \quad h\left(\bar{q}^{2}\right) \equiv \frac{1}{N \sqrt{z}} \ln \left(\frac{\sqrt{z}-1}{\sqrt{z}+1}\right)
$$

Using these quantities the pion mass and the pion decay constant to NNLO are given by

$$
m_{\pi}^{2}=m_{0}\left\{1+x_{2}\left[m_{\pi}\right]_{1}+x_{2}^{2}\left[m_{\pi}\right]_{2}\right\} \quad \text { and } \quad F_{\pi}=F\left\{1+x_{2}\left[F_{\pi}\right]_{1}+x_{2}^{2}\left[F_{\pi}\right]_{2}\right\}
$$


where

$$
\begin{aligned}
{\left[m_{\pi}\right]_{1} } & \equiv 2 \ell_{3}^{r}+\frac{1}{2} L \\
{\left[m_{\pi}\right]_{2} } & \equiv \frac{1}{N}\left(\ell_{1}^{r}+2 \ell_{2}^{r}-\frac{13}{3} L\right)+\frac{163}{96 N^{2}}-\frac{7}{2} k_{1}-2 k_{2}-4\left(\ell_{3}^{r}\right)^{2}+4 \ell_{3}^{r} \ell_{4}^{r} \\
& -\frac{9}{4} k_{3}+\frac{1}{4} k_{4}+r_{m}^{r} \\
{\left[F_{\pi}\right]_{1} } & \equiv \ell_{4}^{r}-L \\
{\left[F_{\pi}\right]_{2} } & \equiv \frac{1}{N}\left(-\frac{1}{2} \ell_{1}^{r}-\ell_{2}^{r}+\frac{29}{12} L\right)-\frac{13}{192 N^{2}}+\frac{7}{4} k_{1}+k_{2}-2 \ell_{3}^{r} \ell_{4}^{r} \\
& +2\left(\ell_{4}^{r}\right)^{2}-\frac{5}{4} k_{4}+r_{f}^{r}
\end{aligned}
$$

Here $r_{m}^{r}$ and $r_{f}^{r}$ are constants stemming from the $\mathcal{O}\left(p^{6}\right)$ Lagrangian after minimal subtraction and $F$ is the pion decay constant in the chiral limit. For the form factor the expansion is written as

$$
f_{\pi \pi}\left(\bar{q}^{2}\right)=1+x_{2}\left[f_{\pi \pi}\right]_{1}+x_{2}^{2}\left(P_{V}^{(2)}+U_{V}^{(2)}\right) .
$$

Here $\left[f_{\pi \pi}\right]_{1}$ is given by

$$
\left[f_{\pi \pi}\right]_{1} \equiv \frac{1}{6}\left(\bar{q}^{2}-4\right) J\left(\bar{q}^{2}\right)+\bar{q}^{2}\left(-\ell_{6}^{r}-\frac{1}{6} L-\frac{1}{18 N}\right)
$$

and $P_{V}^{(2)}$ and $U_{V}^{(2)}$ are the polynomial and dispersive NNLO piece respectively, given by

$$
\begin{aligned}
& P_{V}^{(2)}=\bar{q}^{4}\left[\frac{1}{12} k_{1}-\frac{1}{24} k_{2}+\frac{1}{24} k_{6}\right. \\
& \left.+\frac{1}{9 N}\left(\ell_{1}^{r}-\frac{1}{2} \ell_{2}^{r}+\frac{1}{2} \ell_{6}^{r}-\frac{1}{12} L-\frac{1}{384}-\frac{47}{192 N}\right)+r_{V 2}^{r}\right] \\
& +\bar{q}^{2}\left[-\frac{1}{2} k_{1}+\frac{1}{4} k_{2}-\frac{1}{12} k_{4}+\frac{1}{2} k_{6}-\ell_{4}^{r}\left(2 \ell_{6}^{r}+\frac{1}{9 N}\right)\right. \\
& \left.+\frac{23}{36} \frac{L}{N}+\frac{5}{576 N}+\frac{37}{864 N^{2}}+r_{V 1}^{r}\right] \\
& U_{V}^{(2)}=J\left(\bar{q}^{2}\right)\left[\frac{1}{3} \ell_{1}^{r}\left(-\bar{q}^{4}+4 \bar{q}^{2}\right)+\frac{1}{6} \ell_{2}^{r}\left(\bar{q}^{4}-4 \bar{q}^{2}\right)+\frac{1}{3} \ell_{4}^{r}\left(\bar{q}^{2}-4\right)\right. \\
& +\frac{1}{6} \ell_{6}^{r}\left(-\bar{q}^{4}+4 \bar{q}^{2}\right)-\frac{1}{36} L\left(\bar{q}^{4}+8 \bar{q}^{2}-48\right) \\
& \left.+\frac{1}{N}\left(\frac{7}{108} \bar{q}^{4}-\frac{97}{108} \bar{q}^{2}+\frac{3}{4}\right)\right]+\frac{1}{9} K_{1}\left(\bar{q}^{2}\right) \\
& +\frac{1}{9} K_{2}\left(\bar{q}^{2}\right)\left(\frac{1}{8} \bar{q}^{4}-\bar{q}^{2}+4\right)+\frac{1}{6} K_{3}\left(\bar{q}^{2}\right)\left(\bar{q}^{2}-\frac{1}{3}\right)-\frac{5}{3} K_{4}\left(\bar{q}^{2}\right) \text {. }
\end{aligned}
$$

$r_{V 1}^{r}$ and $r_{V 2}^{r}$ are again coming from the $\mathcal{O}\left(p^{6}\right)$ Lagrangian. 


\section{A.2 Reformulation of ChPT to NNLO for global fits}

For the intended fits as discussed in section 4 it is necessary to reorganize the chiral expansion, since the right hand sides depend on $m_{\pi}$ and $F_{\pi}$ themselves. In this appendix we describe the necessary replacements and list the results.

\section{A.2.1 Conventions and necessary replacements}

For the fits including $f_{\pi \pi}\left(Q^{2}\right)$ it is convenient to define the new fit parameter

$$
\tilde{\ell} \equiv \ell_{1}^{r}-\frac{1}{2} \ell_{2}^{r}
$$

which replaces the fit parameters $\ell_{1}^{r}$ and $\ell_{2}^{r}$ completely in eq. A.10. We also use $\tilde{\ell}$ instead $\ell_{2}^{r}$ in $m_{\pi}^{2}$ and $F_{\pi}$ to have a consistent set of fit parameters.

To make the fit formulae self-consistent we have to replace $m_{\pi}$ and $F_{\pi}$ in each formula with the expressions in eq. A.6 and keep all terms to $\mathcal{O}\left(x_{2}^{2}\right)$. In practice this means we have to replace $m_{\pi}$ and $F_{\pi}$ with its NLO expressions in each NLO term. In the course of this replacement the parameters

$$
x_{2}, \quad L, \quad \bar{q}^{2}, \quad \text { and } \quad J\left(\bar{q}^{2}\right)
$$

are modified. In the results similar parameters appear with the first order parameters $m_{0}$ and $F$ instead of $m_{\pi}^{2}$ and $F_{\pi}$. We thus define:

$$
\begin{array}{rlrl}
\hat{x}_{2} & \equiv \frac{m_{0}}{F^{2}} ; & \hat{q}^{2} & \equiv \frac{q^{2}}{m_{0}} \\
\hat{L} ; \equiv \frac{1}{N} \ln \left(\frac{m_{0}}{\mu^{2}}\right) ; & \hat{\ell}_{i}^{r} \equiv \frac{\gamma_{i}}{2 N}\left(\bar{\ell}_{i}+N \hat{L}\right) ; \\
\hat{k}_{i} \equiv\left(4 \hat{\ell}_{i}^{r}-\gamma_{i} \hat{L}\right) \hat{L} ; & \hat{z} \equiv 1-\frac{4}{\hat{q}^{2}} .
\end{array}
$$

As shorthand notation we further define

$$
\Delta_{m} \equiv 2 \hat{\ell}_{3}^{r}+\frac{1}{2} \hat{L} \quad \text { and } \quad \Delta_{f} \equiv \hat{\ell}_{4}^{r}-\hat{L}
$$

This is convenient, since in the following one just has to set $\Delta_{m}$ and $\Delta_{f}$ to zero to obtain the NNLO formulae of the previous appendix.

Most of the replacements are straight-forward. The only more complicated replacement is the one for the function $J\left(\bar{q}^{2}\right)$, since it is a non-trivial function of $z$. We write the result as

$$
J\left(\bar{q}^{2}\right)=J\left(\hat{q}^{2}\right)-\hat{x}_{2} \frac{\Delta_{m}}{\hat{q}^{2}-4}\left(2 J\left(\hat{q}^{2}\right)-\frac{\hat{q}^{2}}{N}\right) .
$$




\section{A.2.2 Reformulated formulae}

We now list the reformulated formulae for $m_{\pi}^{2}, F_{\pi}$ and $f_{\pi \pi}\left(Q^{2}\right)$. The pion mass is given by

$$
m_{\pi}^{2}=m_{0}\left\{1+\hat{x}_{2}\left[m_{\pi}\right]_{1}+\hat{x}_{2}^{2}\left(\left[m_{\pi}\right]_{2}+\Delta_{m}\left[\Delta_{m}-2 \Delta_{f}\right]+\frac{\Delta_{m}}{2 N}\right)\right\},
$$

where $\left[m_{\pi}\right]_{1}$ and $\left[m_{\pi}\right]_{2}$ are defined as in eq. A.7) with every quantity replaced by its modified version from eq. $(\mathrm{A} .13)$. Similarly the modified ChPT expression for the pion decay constant is

$$
F_{\pi}=F\left\{1+\hat{x}_{2}\left[F_{\pi}\right]_{1}+\hat{x}_{2}^{2}\left(\left[F_{\pi}\right]_{2}+\Delta_{f}\left[\Delta_{m}-2 \Delta_{f}\right]-\frac{\Delta_{m}}{N}\right)\right\},
$$

where again the replacements in $\left[F_{\pi}\right]_{1}$ and $\left[F_{\pi}\right]_{2}$ are implied. For $f_{\pi \pi}\left(\hat{q}^{2}\right)$ we obtain

$$
\begin{aligned}
f_{\pi \pi}\left(\bar{q}^{2}\right) & =1+\hat{x}_{2}\left[f_{\pi \pi}\right]_{1}+\hat{x}_{2}^{2}\left(P_{V}^{(2)}+U_{V}^{(2)}\right) \\
& +\hat{x}_{2}^{2}\left[-\Delta_{m} J\left(\hat{q}^{2}\right)-2 \Delta_{f}\left(\frac{1}{6}\left(\hat{q}^{2}-4\right) J\left(\hat{q}^{2}\right)-\hat{q}^{2}\left[\hat{\ell}_{6}^{r}+\frac{1}{6} \hat{L}+\frac{1}{18 N}\right]\right)\right]
\end{aligned}
$$

with implied replacements in $\left[f_{\pi \pi}\right]_{1}, P_{V}^{(2)}$ and $U_{V}^{(2)}$.

For the pion charge radius as defined in eq. (2.2) the results above yield

$$
\left\langle r_{\pi}^{2}\right\rangle=\frac{1}{m_{0}}\left\{\hat{x}_{2}\left[\left\langle r_{\pi}^{2}\right\rangle\right]_{1}+\hat{x}_{2}^{2}\left(\left[\left\langle r_{\pi}^{2}\right\rangle\right]_{2}-2 \Delta_{F}\left[\left\langle r_{\pi}^{2}\right\rangle\right]_{1}-\frac{\Delta_{m}}{N}\right)\right\}
$$

where $\left[\left\langle r_{\pi}^{2}\right\rangle\right]_{1}$ is the NLO part as given in 18 ,

$$
\left[\left\langle r_{\pi}^{2}\right\rangle\right]_{1}=-\left(6 \hat{\ell}_{6}^{r}+\hat{L}+\frac{1}{N}\right)
$$

and $\left[\left\langle r_{\pi}^{2}\right\rangle\right]_{2}$ the usual NNLO part,

$$
\left[\left\langle r_{\pi}^{2}\right\rangle\right]_{2}=-12 \hat{L} \tilde{\ell}-\frac{1}{2} k_{4}+3 k_{6}-12 \hat{\ell}_{4}^{r} \hat{\ell}_{6}^{r}+\frac{1}{N}\left(-2 \hat{\ell}_{4}^{r}+\frac{31}{6} \hat{L}+\frac{13}{192}-\frac{181}{48 N}\right)+6 r_{V 1}^{r}
$$

Note, that the results discussed above are in agreement with the ones listed in [11].

\section{A.2.3 Inclusion of lattice artefacts}

Since the data indicates the presence of residual lattice artefacts it is desirable to include these effects in the chiral extrapolation. To this end we extend the formulae from the last section of the appendix used for the global fits to the more general form:

$$
\begin{aligned}
\left(m_{\pi}\left[1+\alpha_{m} \frac{a^{2}}{r_{0}^{2}}\right]\right)^{2} & =\mathrm{A} .16 ; \\
F_{\pi} & =\mathrm{A} .17+\alpha_{f} \frac{a^{2}}{r_{0}^{2}} ; \\
f_{\pi \pi}\left(\hat{q}^{2}\right) & =\mathrm{A} .18+\alpha_{r} \frac{a^{2}}{r_{0}^{2}} \frac{m_{0} \hat{q}^{2}}{6} ; \\
\left\langle r_{\pi}^{2}\right\rangle & =\mathrm{A} .19+\alpha_{r} \frac{a^{2}}{r_{0}^{2}} .
\end{aligned}
$$


Note that for $m_{\pi}$ the lattice artefacts are expected to be of $\mathcal{O}\left(a^{2}\right)$ for the mass itself and thus should be included on the left hand side.

\section{References}

[1] M. Hasenbusch, Speeding up the hybrid Monte Carlo algorithm for dynamical fermions, Phys.Lett. B519 (2001) 177-182, hep-lat/0107019.

[2] M. Lüscher, Schwarz-preconditioned HMC algorithm for two-flavour lattice QCD, Comput.Phys.Commun. 165 (2005) 199-220, hep-lat/0409106.

[3] C. Urbach, K. Jansen, A. Shindler, and U. Wenger, HMC algorithm with multiple time scale integration and mass preconditioning, Comput.Phys.Commun. 174 (2006) 87-98, hep-lat/0506011.

[4] M. Clark and A. Kennedy, Accelerating dynamical fermion computations using the rational hybrid Monte Carlo (RHMC) algorithm with multiple pseudofermion fields, Phys.Rev.Lett. 98 (2007) 051601, hep-lat/0608015].

[5] M. Lüscher, Local coherence and deflation of the low quark modes in lattice QCD, JHEP 0707 (2007) 081, 0706.2298.

[6] M. Lüscher, Deflation acceleration of lattice QCD simulations, JHEP 0712 (2007) 011, 0710.5417 .

[7] M. Lüscher and F. Palombi, Fluctuations and reweighting of the quark determinant on large lattices, PoS LATTICE2008 (2008) 049, 0810.0946.

[8] M. Marinkovic and S. Schaefer, Comparison of the mass preconditioned HMC and the $D D$-HMC algorithm for two-flavour QCD, PoS LATTICE2010 (2010) 031, 1011.0911.

[9] G. Colangelo, S. Dürr, A. Jüttner, L. Lellouch, H. Leutwyler, et al., Review of lattice results concerning low energy particle physics, Eur.Phys.J. C71 (2011) 1695, 1011.4408.

[10] QCDSF and UKQCD Collaboration, D. Brömmel et al., The Pion form-factor from lattice QCD with two dynamical flavours, Eur.Phys.J. C51 (2007) 335-345, hep-lat/0608021.

[11] ETM Collaboration, R. Frezzotti, V. Lubicz, and S. Simula, Electromagnetic form factor of the pion from twisted-mass lattice $Q C D$ at $N(f)=2$, Phys.Rev. D79 (2009) 074506, 0812.4042.

[12] P. Boyle, J. Flynn, A. Jüttner, C. Kelly, H. de Lima, et al., The Pion's electromagnetic form-factor at small momentum transfer in full lattice QCD, JHEP 0807 (2008) 112, 0804.3971. 
[13] JLQCD and TWQCD Collaboration, S. Aoki et al., Pion form factors from two-flavor lattice QCD with exact chiral symmetry, Phys.Rev. D80 (2009) 034508, 0905.2465 .

[14] O. H. Nguyen, K.-I. Ishikawa, A. Ukawa, and N. Ukita, Electromagnetic form factor of pion from $N_{f}=2+1$ dynamical flavor QCD, JHEP 1104 (2011) 122, 1102.3652.

[15] B. B. Brandt, A. Jüttner, and H. Wittig, Calculation of the pion electromagnetic form factor from lattice $Q C D, 1109.0196$.

[16] J. Gasser and H. Leutwyler, Chiral Perturbation Theory: Expansions in the Mass of the Strange Quark, Nucl.Phys. B250 (1985) 465.

[17] J. Gasser and H. Leutwyler, Low-Energy Expansion of Meson Form-Factors, Nucl.Phys. B250 (1985) 517-538.

[18] J. Bijnens, G. Colangelo, and P. Talavera, The Vector and scalar form-factors of the pion to two loops, JHEP 9805 (1998) 014, hep-ph/9805389.

[19] J. Bijnens and P. Talavera, Pion and kaon electromagnetic form-factors, JHEP 0203 (2002) 046, hep-ph/0203049.

[20] C. Aubin and T. Blum, Calculating the hadronic vacuum polarization and leading hadronic contribution to the muon anomalous magnetic moment with improved staggered quarks, Phys.Rev. D75 (2007) 114502, hep-lat/0608011.

[21] X. Feng, K. Jansen, M. Petschlies, and D. B. Renner, Two-flavor QCD correction to lepton magnetic moments at leading-order in the electromagnetic coupling, Phys.Rev.Lett. 107 (2011) 081802, 1103.4818.

[22] P. Boyle, L. Del Debbio, E. Kerrane, and J. Zanotti, Lattice Determination of the Hadronic Contribution to the Muon g-2 using Dynamical Domain Wall Fermions, Phys.Rev. D85 (2012) 074504, 1107.1497.

[23] M. Della Morte and A. Jüttner, Quark disconnected diagrams in chiral perturbation theory, JHEP 1011 (2010) 154, 1009.3783.

[24] M. Della Morte, B. Jäger, A. Jüttner, and H. Wittig, Towards a precise lattice determination of the leading hadronic contribution to $(g-2)_{\mu}$, JHEP 1203 (2012) 055, 1112.2894 .

[25] P. F. Bedaque, Aharonov-Bohm effect and nucleon nucleon phase shifts on the lattice, Phys.Lett. B593 (2004) 82-88, nucl-th/0402051.

[26] G. de Divitiis, R. Petronzio, and N. Tantalo, On the discretization of physical momenta in lattice QCD, Phys.Lett. B595 (2004) 408-413, hep-lat/0405002.

[27] C. Sachrajda and G. Villadoro, Twisted boundary conditions in lattice simulations, Phys.Lett. B609 (2005) 73-85, hep-lat/0411033. 
[28] P. Boyle, J. Flynn, A. Jüttner, C. Sachrajda, and J. Zanotti, Hadronic form factors in Lattice QCD at small and vanishing momentum transfer, JHEP 0705 (2007) 016, hep-lat/0703005.

[29] B. B. Brandt, S. Capitani, D. Djukanovic, G. von Hippel, B. Jäger, et al., Wilson fermions at fine lattice spacings: scale setting, pion form factors and $(g-2)_{\mu}$, PoS LATTICE2010 (2010) 164, 1010.2390.

[30] B. Brandt, S. Capitani, M. Della Morte, D. Djukanovic, J. Gegelia, et al., Form factors in lattice QCD, Eur.Phys.J.ST 198 (2011) 79-94, 1106.1554.

[31] B. B. Brandt, A. Jüttner, and H. Wittig, The electromagnetic form factor of the pion in two-flavour lattice QCD, PoS ConfinementX (2012) 112, [1301.3513].

[32] UKQCD Collaboration, J. Flynn, A. Jüttner, and C. Sachrajda, A Numerical study of partially twisted boundary conditions, Phys.Lett. B632 (2006) 313-318, hep-lat/0506016.

[33] K. G. Wilson, Confinement of Quarks, Phys.Rev. D10 (1974) 2445-2459.

[34] ALPHA Collaboration, K. Jansen and R. Sommer, $O(a)$ improvement of lattice QCD with two flavors of Wilson quarks, Nucl.Phys. B530 (1998) 185-203, hep-lat/9803017.

[35] M. Lüscher, S. Sint, R. Sommer, and P. Weisz, Chiral symmetry and O(a) improvement in lattice QCD, Nucl.Phys. B478 (1996) 365-400, hep-lat/9605038.

[36] M. Della Morte, R. Hoffmann, and R. Sommer, Non-perturbative improvement of the axial current for dynamical Wilson fermions, JHEP 0503 (2005) 029, hep-lat/0503003.

[37] S. Sint and P. Weisz, Further one-loop results in $O(a)$ improved lattice QCD, Nucl. Phys. Proc. Suppl. 63 (1998) 856-858, hep-lat/9709096.

[38] P. Fritzsch, J. Heitger, and N. Tantalo, Non-perturbative improvement of quark mass renormalization in two-flavour lattice QCD, JHEP 1008 (2010) 074, 1004.3978.

[39] P. Fritzsch, F. Knechtli, B. Leder, M. Marinkovic, S. Schaefer, et al., The strange quark mass and Lambda parameter of two flavor QCD, Nucl.Phys. B865 (2012) 397-429, 1205.5380.

[40] M. Della Morte, R. Sommer, and S. Takeda, On cutoff effects in lattice QCD from short to long distances, Phys.Lett. B672 (2009) 407-412, 0807.1120.

[41] ALPHA Collaboration, M. Della Morte et al., Non-perturbative quark mass renormalization in two-flavor QCD, Nucl.Phys. B729 (2005) 117-134, hep-lat/0507035. 
[42] L. Del Debbio, L. Giusti, M. Lüscher, R. Petronzio, and N. Tantalo, QCD with light Wilson quarks on fine lattices. II. DD-HMC simulations and data analysis, JHEP 0702 (2007) 082, hep-lat/0701009.

[43] Particle Data Group Collaboration, K. Nakamura et al., Review of particle physics, J.Phys.G G37 (2010) 075021.

[44] J. Bulava, M. Donnellan, and R. Sommer, On the computation of hadron-to-hadron transition matrix elements in lattice QCD, JHEP 1201 (2012) 140, 1108.3774].

[45] S. Capitani, M. Della Morte, G. von Hippel, B. Jäger, A. Jüttner, et al., The nucleon axial charge from lattice QCD with controlled errors, Phys.Rev. D86 (2012) 074502, 1205.0180.

[46] R. Sommer, A New way to set the energy scale in lattice gauge theories and its applications to the static force and $\alpha_{s}$ in SU(2) Yang-Mills theory, Nucl.Phys. B411 (1994) 839-854, hep-lat/9310022.

[47] ALPHA Collaboration, B. Leder and F. Knechtli, Scale $r_{0}$ and the static potential from the CLS lattices, PoS LATTICE2010 (2010) 233, 1012.1141.

[48] S. Capitani, M. Della Morte, G. von Hippel, B. Knippschild, and H. Wittig, Scale setting via the $\Omega$ baryon mass, PoS LATTICE2011 (2011) 145, 1110.6365.

[49] UKQCD Collaboration, M. Foster and C. Michael, Quark mass dependence of hadron masses from lattice QCD, Phys.Rev. D59 (1999) 074503, hep-lat/9810021.

[50] UKQCD Collaboration, C. McNeile and C. Michael, Decay width of light quark hybrid meson from the lattice, Phys.Rev. D73 (2006) 074506, hep-lat/0603007.

[51] P. Boyle, A. Jüttner, C. Kelly, and R. Kenway, Use of stochastic sources for the lattice determination of light quark physics, JHEP 0808 (2008) 086, 0804.1501.

[52] E. Endress, A. Jüttner, and H. Wittig, On the efficiency of stochastic volume sources for the determination of light meson masses, 1111.5988.

[53] P. Boyle, J. Flynn, A. Jüttner, C. Kelly, C. Maynard, et al., $K \rightarrow \pi$ form factors with reduced model dependence, Eur.Phys.J. C69 (2010) 159-167, 1004.0886.

[54] B. Efron, Bootstrap methods: another look at the jacknife, Ann. Statist. 7 (1979) 1-26.

[55] C. Michael, Fitting correlated data, Phys.Rev. D49 (1994) 2616-2619, hep-lat/9310026.

[56] F.-J. Jiang and B. Tiburzi, Flavor twisted boundary conditions, pion momentum, and the pion electromagnetic form-factor, Phys.Lett. B645 (2007) 314-321, hep-lat/0610103.

[57] NA7 Collaboration, S. Amendolia et al., A Measurement of the Space - Like Pion Electromagnetic Form-Factor, Nucl.Phys. B277 (1986) 168. 
[58] G. Colangelo, S. Dürr, and C. Haefeli, Finite volume effects for meson masses and decay constants, Nucl.Phys. B721 (2005) 136-174, hep-lat/0503014.

[59] F.-J. Jiang and B. C. Tiburzi, Flavor Twisted Boundary Conditions in the Breit Frame, Phys.Rev. D78 (2008) 037501, 0806.4371.

[60] G. Colangelo, J. Gasser, and H. Leutwyler, $\pi \pi$ scattering, Nucl.Phys. B603 (2001) 125-179, hep-ph/0103088.

[61] J. Gasser and H. Leutwyler, Chiral Perturbation Theory to One Loop, Annals Phys. 158 (1984) 142.

[62] U. Burgi, Charged pion pair production and pion polarizabilities to two loops, Nucl.Phys. B479 (1996) 392-426, hep-ph/9602429].

[63] U. Burgi, Charged pion polarizabilities to two loops, Phys.Lett. B377 (1996) 147-152, hep-ph/9602421.

[64] J. Bijnens, G. Colangelo, G. Ecker, J. Gasser, and M. Sainio, Pion pion scattering at low-energy, Nucl.Phys. B508 (1997) 263-310, hep-ph/9707291.

[65] L. Del Debbio, H. Panagopoulos, and E. Vicari, theta dependence of $S U(N)$ gauge theories, JHEP 0208 (2002) 044, hep-th/0204125.

[66] S. Schaefer, R. Sommer, and F. Virotta, Investigating the critical slowing down of QCD simulations, PoS LAT2009 (2009) 032, 0910.1465.

[67] ALPHA Collaboration, S. Schaefer, R. Sommer, and F. Virotta, Critical slowing down and error analysis in lattice QCD simulations, Nucl.Phys. B845 (2011) 93-119, 1009.5228.

[68] M. Lüscher and S. Schaefer, Lattice QCD without topology barriers, JHEP 1107 (2011) $036,1105.4749$.

[69] M. Lüscher and S. Schaefer, Lattice QCD with open boundary conditions and twisted-mass reweighting, Comput.Phys.Commun. 184 (2013) 519-528, 1206.2809.

[70] M. Gell-Mann, R. Oakes, and B. Renner, Behavior of current divergences under SU(3) $x$ SU(3), Phys.Rev. 175 (1968) 2195-2199. 